SLAC-PUB-10680

hep-ph/yymmnnn

August 30, 2004

\title{
Loop Corrections to Heavy-to-Light Form Factors and Evanescent Operators in SCET
}

\author{
Thomas Becher and Richard J. Hill \\ Stanford Linear Accelerator Center, Stanford University \\ Stanford, CA 94309, U.S.A.
}

\begin{abstract}
One-loop matching corrections are calculated for Soft-Collinear Effective Theory (SCET) operators relevant to the analysis of heavy-to-light meson form factors at large recoil. The numerical impact of radiative corrections on form factor predictions is assessed. Evanescent operators in the effective theory are studied and it is shown that even in problems of the Sudakov type, these operators can be renormalized to have vanishing matrix elements.
\end{abstract}

Submitted to Journal of High Energy Physics 


\section{Introduction}

The methods that were used to establish factorization theorems for high-energy QCD processes can also be applied to study decays of $B$-mesons into light hadrons. In this case the hard scale is set by the mass of the heavy $b$-quark and factorization theorems for inclusive $[1,2,3]$ as well as exclusive [4] $B$-decays to light hadrons arise in the heavy-quark limit. Soft-Collinear Effective Theory (SCET) is the effective field theory arising in this limit. It describes the heavy-quark expansion for these decays, and permits the study of their factorization and renormalization properties $[5,6,7,8,9]$.

Semileptonic decays, such as $B \rightarrow \pi \ell \nu$, are the simplest exclusive heavy-to-light meson processes. These decays are described by weak-interaction form factors, which at large recoil energies of the light meson, $E \sim m_{B} / 2 \gg \Lambda_{\mathrm{QCD}}$, take the form [10],

$$
F_{i}^{B \rightarrow M}(E)=C_{i}(E) \zeta_{M}(E)+\int_{0}^{\infty} \frac{d \omega}{\omega} \phi_{B}(\omega) \int_{0}^{1} d u f_{M} \phi_{M}(u) T_{i}(E, \omega, u),
$$

up to corrections suppressed by $\Lambda_{\mathrm{QCD}} / m_{b}$. Here $M$ denotes the final state (pseudoscalar or vector) meson. The process-dependent Wilson coefficient functions $C_{i}(E)$ and $T_{i}(E, \omega, u)$ are calculable in perturbation theory and are the subject of the present paper. The wave functions, or more precisely, light cone distribution amplitudes (LCDAs) $\phi_{B}(\omega)$ and $\phi_{M}(u)$, and the functions $\zeta_{M}(E)$, are process-independent nonperturbative hadronic parameters. The factorization theorem (1) has recently been established in the context of SCET $[11,12,13]$.

At large recoil, the form factors $F_{i}^{B \rightarrow M}$ are in fact subleading quantities, requiring powersuppressed interactions to mediate the $B \rightarrow M$ transition. Since the light degrees of freedom inside the $B$-meson carry soft momenta, $p^{\mu} \sim \Lambda_{\mathrm{QCD}}$, whereas the degrees of freedom inside the final-state meson typically share the large energy of the meson, the transition can only occur if: (i), the partons are in an atypical "endpoint" configuration, allowing the soft $B$ meson constituents to be absorbed into the energetic light meson; or (ii), a large momentum is exchanged between the active quark and the light spectator degrees of freedom. Possibility (i), the so-called soft-overlap mechanism, is described by the non-factorizable part $\zeta_{M}(E)$ of the form factors, and in the effective theory is given by the matrix element of the leadingorder heavy-to-light quark current. This current involves only the two-component spinor fields of SCET and Heavy-Quark Effective Theory (HQET) [14], and mediates the transition of the heavy quark inside the $B$-meson into an energetic light quark component of the light meson. The simple spin structure of the two-component fields results in "large-energy" spin symmetries, such that a single function $\zeta_{M}(E)$ describes the soft-overlap contribution to all form factors involving the same final-state meson $M$ [15]. Possibility (ii), the hard-scattering mechanism, results from subleading effective theory current operators involving an additional gluon field transferring large momentum to the spectator. Their matrix elements factorize and correspond to the second term in equation (1).

The form factors receive contributions associated with different momentum scales: the hard scale $\mu^{2} \sim m_{b}^{2}$, an intermediate scale $\mu^{2} \sim m_{b} \Lambda_{\mathrm{QCD}}$ and the soft scale $\mu^{2} \sim \Lambda_{\mathrm{QCD}}^{2}$. In order to perform a renormalization-group ( $\mathrm{RG}$ ) resummation of large perturbative logarithms involving ratios of these scales, a two-step matching is required, first from QCD onto an intermediate theory, denoted $\mathrm{SCET}_{\mathrm{I}}$, and then from $\mathrm{SCET}_{\mathrm{I}}$ onto the final low-energy theory, denoted 
QCD
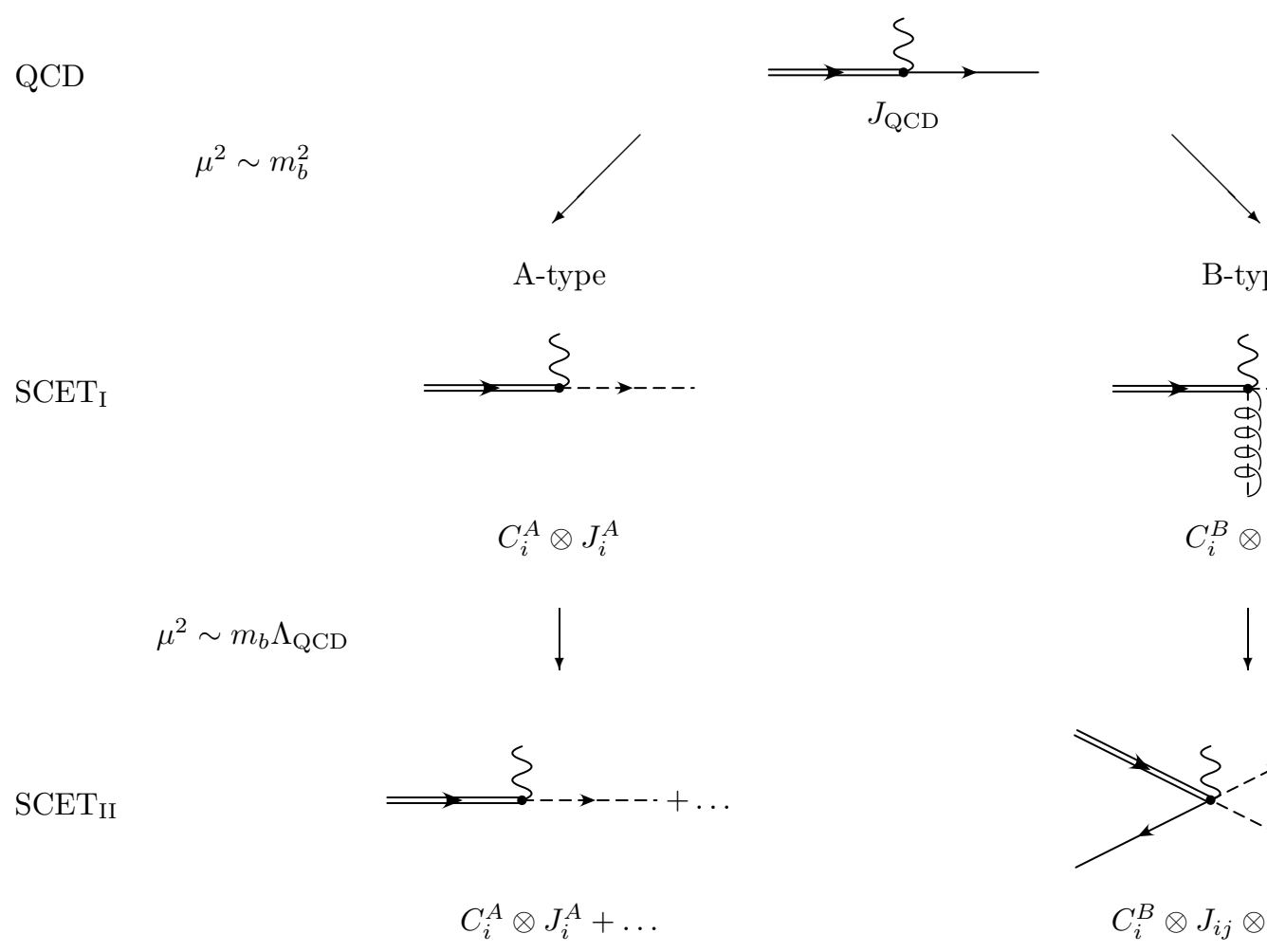

B-type

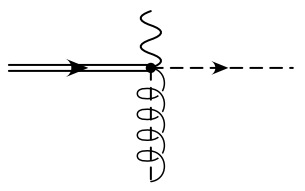

$C_{i}^{B} \otimes J_{i}^{B}$

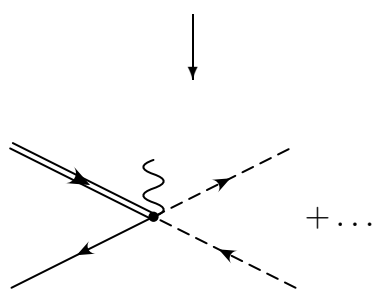

$C_{i}^{B} \otimes J_{i j} \otimes O_{j}+\ldots$

Figure 1: Two step matching from QCD to $\mathrm{SCET}_{\mathrm{I}}$ to $\mathrm{SCET}_{\mathrm{II}}$. The double line denotes the heavy quark; dashed lines denote hard-collinear fields in $\mathrm{SCET}_{\mathrm{I}}$ and collinear fields in $\mathrm{SCET}_{\mathrm{II}}$. The symbol " $\otimes$ " indicates the convolution of the Wilson coefficient functions with the non-local operators.

$\mathrm{SCET}_{\mathrm{II}}$; see Figure 1. For the leading-power currents defining the soft-overlap contributions, the matching from QCD onto $\mathrm{SCET}_{\mathrm{I}}$ has been performed through one-loop order in [5], and the subsequent matching onto $\mathrm{SCET}_{\mathrm{II}}$ is given in [13]. Resummation of large logarithms in the coefficient functions $C_{i}(E)$ results in a universal $\mathrm{RG}$ factor $[5,13]$, the same for all form factors. However, the numerical value of this universal factor is presently of limited utility, since the functions $\zeta_{M}(E)$ are not known at any fixed renormalization scale. On the other hand, some predictions exist for the hadronic wavefunctions appearing in the second term of (1), and we may study the numerical impact of renormalization-group evolution down to hadronic scales where these predictions are reliable. In a recent paper in collaboration with Lee and Neubert [16], we resummed all leading single and double logarithms in the hard scattering kernels $T_{i}(E, \omega, u)$. At leading order in RG-improved perturbation theory, the resummed result is obtained using tree-level matching. However, since $\alpha_{s}$ is sizeable at the relevant scales, we expect one-loop matching corrections to also be important. In [16] the size of these matching corrections was investigated using the scalar current as an example, and they were found to be comparable to the resummation effects. In the present paper, we compute the complete one-loop matching results for the hard-scattering term, for both matching steps.

The outline of the paper is as follows. In Section 2 we give the matching coefficients for $\mathrm{QCD}$ vector and tensor currents onto the subleading $\mathrm{SCET}_{\mathrm{I}}$ operators relevant to the 
hard-scattering form factor contributions. The coefficients for the scalar current have been calculated previously $[16,17]$, and can in fact be derived from the vector current results [16]. The vector and tensor coefficients have also been calculated by Beneke, Kiyo and Yang [17] and we find full agreement with their results; in Section 2 we list only those coefficients necessary for $B \rightarrow M$ form factors, in the "primed" operator basis of [16]. The Wilson coefficients, also called jet functions, that arise in the second matching step, from SCET I onto $\mathrm{SCET}_{\mathrm{II}}$, are considered in Section 3. We have already stated the results relevant to the form factors in [16]. Here we present the general expressions from which those results were derived, and provide details of the calculation.

For dimension $d=4-2 \epsilon$ in dimensional regularization, so-called evanescent operators [18, $19,20]$ arise in the matching of QCD onto SCET. The matrix elements of these operators vanish at tree-level for $d=4$, but in a generic renormalization scheme they are nonzero at higher order. To avoid introducing new hadronic functions at each order in perturbation theory to parameterize the matrix elements of the evanescent operators, it is important to show that a renormalization scheme exists for which these operators vanish exactly in four dimensions. The existence of such a scheme is related to the technical observation that in loop Feynman diagrams involving an evanescent operator, a contribution proportional to a physical operator contains a factor $\epsilon$ from Dirac algebra, which cancels a $1 / \epsilon$ divergence arising from integration over loop momenta; the resulting finite contribution is local, and so may be subtracted by a suitable counterterm. It can also be shown that in this scheme no mixing occurs of the physical into the evanescent operators, and thus the matching coefficients onto renormalized evanescent operators are irrelevant to the calculation of physical matrix elements. In processes involving Sudakov double logarithms, the existence of $1 / \epsilon^{2}$ divergences in loop integrations poses a potential obstacle to these standard arguments. In Section 4 we show that despite this complication, renormalization schemes exist in which the matrix elements of renormalized evanescent operators vanish. This section also discusses a related issue, the choice of evanescent operators. Different choices correspond to different renormalization schemes for the physical operators. For the four-quark SCET II operators describing the hard-scattering form factor contributions, we isolate the particular operator basis which corresponds to the $\overline{\mathrm{MS}}$ scheme after Fierz transformation. Upon taking heavy-light meson matrix elements, this ensures that the LCDAs appearing in (1) are renormalized in the $\overline{\mathrm{MS}}$ scheme.

The phenomenological importance of one-loop matching corrections to heavy-to-light form factors is investigated in Section 5. Hard-scale corrections, corresponding to QCD $\rightarrow \mathrm{SCET}_{\mathrm{I}}$ matching, are found to be of order $10-20 \%$. These corrections determine the size of violations to the large-energy spin-symmetry relations obeyed by the soft-overlap term, and also the size of non-universal corrections to the hard-scattering term. We notice that in the soft-overlap case, the radiative corrections for different form factors are remarkably similar, and therefore have little effect on the symmetry relations. The numerical impact of one-loop contributions to the jet functions for $\mathrm{SCET}_{\mathrm{I}} \rightarrow \mathrm{SCET}_{\mathrm{II}}$ matching is more difficult to estimate, owing to the lack of precise knowledge on the $B$-meson wavefunction. However, when hard-scale QCD $\rightarrow$ $\mathrm{SCET}_{\mathrm{I}}$ matching corrections are ignored, the hard-scattering contributions are described by the universal functions $H_{M}(E)$ introduced in [16]; radiative corrections to the jet functions are therefore not relevant to the conclusions which can be drawn based solely on this universality. The radiative corrections are necessary in order to relate the functions $H_{M}(E)$ to models for 
the $B$-meson wavefunction, and we investigate the size of the corrections for two such models, finding that they increase $H_{M}(E)$ by $\sim 20-30 \%$. Section 6 provides a discussion and our conclusions.

\section{One-loop matching from QCD onto SCET $_{\mathrm{I}}$}

In this section, we deal with $\mathrm{SCET}_{\mathrm{I}}$, which describes the interaction of energetic light particles of virtuality $p^{2} \sim m_{b} \Lambda_{\mathrm{QCD}}$ with a static heavy quark. The soft sector of $\mathrm{SCET}_{\mathrm{I}}$ is described by HQET, with a heavy quark of velocity $v$. Different components of hard-collinear momenta and fields scale with different powers of the expansion parameter $\lambda^{1 / 2}=\left(\Lambda_{\mathrm{QCD}} / m_{b}\right)^{1 / 2}$. The large momentum components are isolated to obtain a definite power counting by working with light-like reference vectors, $n^{\mu}$ in the direction of the jet of outgoing collinear particles, and $\bar{n}^{\mu}$ a complementary vector satisfying $n \cdot \bar{n}=2$ :

$$
p^{\mu}=n \cdot p \frac{\bar{n}^{\mu}}{2}+\bar{n} \cdot p \frac{n^{\mu}}{2}+p_{\perp}^{\mu} .
$$

The components $\left(n \cdot p, \bar{n} \cdot p, p_{\perp}\right)$ of a hard-collinear momentum are defined to scale as $\left(\lambda, 1, \lambda^{1 / 2}\right)$, while for a soft momentum the components scale as $(\lambda, \lambda, \lambda)$. We choose $\bar{n}^{\mu}$ such that $v_{\perp}=0$, implying $\bar{n}^{\mu}=\left(2 v^{\mu}-n^{\mu} / n \cdot v\right) / n \cdot v$. The canonical choices are $v^{\mu}=(1,0,0,0), n^{\mu}=(1,0,0,1)$ and $\bar{n}^{\mu}=(1,0,0,-1)$, corresponding to the energetic jet in the $z$-direction as viewed from the rest-frame of the heavy meson. Throughout this paper we use the notation and conventions of [16], to which we refer the reader for details and references.

We now consider the effective theory representation of the scalar, vector and tensor flavorchanging currents,

$$
S=\bar{q} b, \quad V^{\mu}=\bar{q} \gamma^{\mu} b, \quad T^{\mu \nu}=(-i) \bar{q} \sigma^{\mu \nu} b=\bar{q} \gamma^{[\mu} \gamma^{\nu]} b .
$$

We use square brackets around indices to denote antisymmetrization. The QCD scalar and tensor currents require renormalization. We work in dimensional regularization for $d=4-2 \epsilon$ dimensions, and define the QCD as well as the effective theory operators in the modified minimal subtraction $(\overline{\mathrm{MS}})$ scheme. ${ }^{1}$ We use the naive dimensional regularization (NDR) scheme for $\gamma_{5}$ so that the pseudoscalar and pseudovector currents renormalize identically to the scalar and vector currents, and our results for the matching are independent of the chirality of the light quark $q$ in the current operator. The construction of the effective theory currents is discussed in [16]. For the case of the scalar current, which we take at position $x=0$, only two effective theory operators are needed through the first subleading order:

$$
\begin{gathered}
J_{S}^{A}(s, x=0)=\bar{X}_{h c}(s \bar{n})\left(1-\frac{i \overleftarrow{\not} \perp}{i \bar{n} \cdot \overleftarrow{\partial}} \frac{\not h}{2}\right) h(0), \\
J_{S}^{B}(s, r, x=0)=\bar{X}_{h c}(s \bar{n}) \mathcal{A}_{h c \perp}(r \bar{n}) h(0) .
\end{gathered}
$$

\footnotetext{
${ }^{1}$ In Section 4, we consider the modifications to this scheme necessary to ensure the vanishing of renormalized evanescent operators.
} 
The field $h$ is the heavy-quark field, $X_{h c}=W_{h c}^{\dagger} \xi_{h c}$ is the hard-collinear quark field multiplied by a hard-collinear Wilson line $W_{h c}$ in the $\bar{n}$-direction, and $\mathcal{A}_{h c \perp}^{\mu}=W_{h c}^{\dagger}\left[\left(i \partial_{\perp}^{\mu}+A_{h c \perp}^{\mu}\right) W_{h c}\right]$, where $A_{h c}$ is the hard-collinear gluon field. Note that the above operators are non-local: the hard-collinear fields live at different points on the light-ray in the $\bar{n}$-direction. This is a consequence of the fact that $\bar{n} \cdot \partial$ derivatives on hard-collinear fields count as quantities of order one, so that operators involving arbitrary functions of such derivatives may appear at a given order in the power counting. The Wilson coefficients of these operators are then functions of the position arguments and the representation of the scalar current at $x=0$ in the effective theory reads

$$
\begin{aligned}
\bar{q} b & \rightarrow \int d s \tilde{C}_{S}^{A}(s) J_{S}^{A}(s)+\frac{1}{2 E} \int d r d s \tilde{C}_{S}^{B}(s, r) J_{S}^{B}(s, r)+\ldots \\
& =C_{S}^{A}(E) J_{S}^{A}(0)+\frac{1}{2 E} \int d u C_{S}^{B}(E, u) J_{S}^{B}(u)+\ldots
\end{aligned}
$$

where the factor $1 / 2 E$ has been inserted into the second term for convenience. We use a tilde to denote the coefficients in position space. The momentum space coefficient functions in the second line are defined as

$$
\begin{aligned}
C_{S}^{A}(E) & =\int d s e^{i s \bar{n} \cdot P} \tilde{C}_{S}^{A}(s) \\
C_{S}^{B}(E, u) & =\int d r d s e^{i(u s+\bar{u} r) \bar{n} \cdot P} \tilde{C}_{S}^{B}(s, r) .
\end{aligned}
$$

Here $P=P_{\text {out }}-P_{\text {in }}$ is the total hard-collinear momentum of external states, and $E \equiv$ $(n \cdot v)(\bar{n} \cdot P) / 2$. The variable $u \in[0,1]$ denotes the fraction of the energy $E$ carried by the quark field $\bar{X}_{h c}$ and $\bar{u}=1-u$ the fraction carried by $\mathcal{A}_{h c \perp}^{\mu}$.

The fact that the subleading term in $J_{S}^{A}$ involving a perpendicular partial derivative has the same Wilson coefficient as the leading order term is a consequence of reparametrization invariance, the fact that QCD is independent of the reference vectors $n_{\mu}$ and $v_{\mu}$ used in the construction of the effective theory $[7,21,16]$. Due to the presence of the reference vectors $n_{\mu}$ and $v_{\mu}$, the vector and tensor currents are represented by several effective theory operators, even at leading order. However the general structure of the subleading currents is the same as in the scalar case: $A$-type operators involve perpendicular partial derivatives and have the same Wilson coefficient as the leading order operators; the remaining, $B$-type operators involve the insertion of an additional perpendicular gluon field $\mathcal{A}_{h c \perp}^{\mu}$. The complete basis of $\mathrm{SCET}_{\mathrm{I}}$ current operators can be found e.g. in [16]. In that reference two different sets of basis operators are used, $J_{i}^{B}$ and $J_{i}^{B^{\prime}}$. Here we use the $B$-type operators in the primed basis, which are more convenient in that they renormalize multiplicatively and match in a simple way onto SCET $_{\text {II }}$. Suppressing the " $h c$ " subscript on hard-collinear fields, the vector current operators in this basis are

$$
\begin{aligned}
J_{V 1}^{B^{\prime} \mu} & =\bar{X}(s \bar{n}) \mathcal{A}_{\perp}(r \bar{n}) \gamma_{\perp}^{\mu} h(0), & J_{V 2}^{B^{\prime} \mu} & =\bar{X}(s \bar{n}) \mathcal{A}_{\perp}(r \bar{n}) v^{\mu} h(0), \\
J_{V 3}^{B^{\prime} \mu} & =\bar{X}(s \bar{n}) \mathcal{A}_{\perp}(r \bar{n}) \frac{n^{\mu}}{n \cdot v} h(0), & J_{V 4}^{B^{\prime} \mu} & =\bar{X}(s \bar{n}) \gamma_{\perp}^{\mu} \mathcal{A}_{\perp}(r \bar{n}) h(0),
\end{aligned}
$$



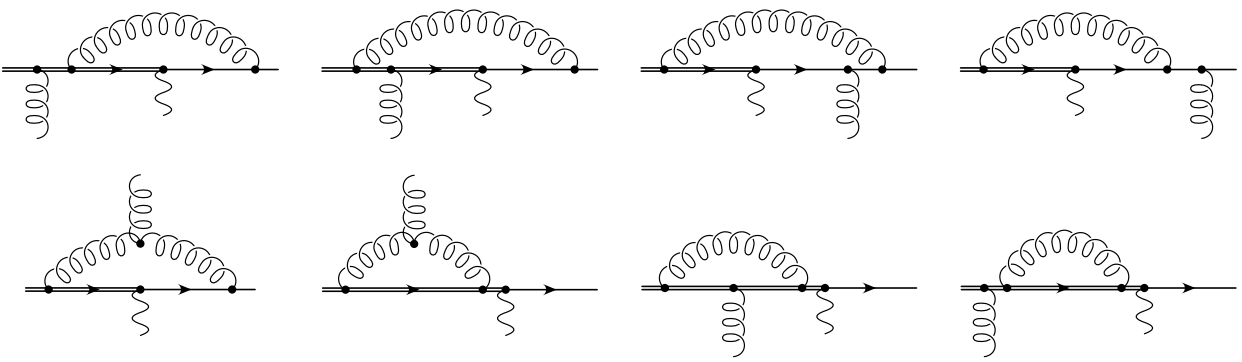

Figure 2: One-loop QCD diagrams contributing to the matching calculation for the subleading scalar current. The double line denotes the heavy quark.

and the tensor current is represented by

$$
\begin{array}{rlrl}
J_{T 1}^{B^{\prime} \mu \nu} & =\bar{X}(s \bar{n}) \mathcal{A}_{\perp}(r \bar{n}) \gamma_{\perp}^{[\mu} \gamma_{\perp}^{\nu]} h(0), & & J_{T 2}^{B^{\prime} \mu \nu}=\bar{X}(s \bar{n}) \mathcal{A}_{\perp}(r \bar{n}) v^{[\mu} \gamma_{\perp}^{\nu]} h(0), \\
J_{T 3}^{B^{\prime} \mu \nu}=\bar{X}(s \bar{n}) \mathcal{A}_{\perp}(r \bar{n}) \frac{n^{[\mu} \gamma_{\perp}^{\nu]}}{n \cdot v} h(0), & & J_{T 4}^{B^{\prime} \mu \nu}=\bar{X}(s \bar{n}) \mathcal{A}_{\perp}(r \bar{n}) \frac{n^{[\mu} v^{\nu]}}{n \cdot v} h(0), \\
J_{T 5}^{B^{\prime} \mu \nu}=\bar{X}(s \bar{n}) \mathcal{A}_{\perp \alpha}(r \bar{n}) \gamma_{\perp}^{[\alpha} \gamma_{\perp}^{\mu} \gamma_{\perp}^{\nu]} h(0), & & J_{T 6}^{B^{\prime} \mu \nu}=\bar{X}(s \bar{n}) v^{[\mu} \gamma_{\perp}^{\nu]} \mathcal{A}_{\perp}(r \bar{n}) h(0), \\
J_{T 7}^{B^{\prime} \mu \nu}=\bar{X}(s \bar{n}) \frac{n^{[\mu} \gamma_{\perp}^{\nu]}}{n \cdot v} \mathcal{A}_{\perp}(r \bar{n}) h(0) . &
\end{array}
$$

One-loop matching corrections to the leading order, A-type effective theory currents were evaluated in [5] and the results were confirmed by the findings of [17]. Here, we focus on the Wilson coefficients of the $B$-type operators which can be obtained by evaluating the QCD diagrams shown in Figure 2. By performing the matching on-shell, one can avoid the calculation of effective theory loop diagrams, as discussed in [16], where we presented the one-loop matching for the scalar current. Only six of the above currents match onto $\mathrm{SCET}_{\mathrm{II}}$ four-quark operators relevant for the heavy-to-light form factors and we now give the results for those six Wilson coefficients. Introducing $x=2 \mathrm{E} / \mathrm{m}_{b}$, we find for the case of the vector current,

$$
\begin{aligned}
C_{V 2}^{B^{\prime}}=-2+ & \frac{C_{F} \alpha_{s}}{4 \pi}\left\{4 \ln ^{2} \frac{2 E}{\mu}-2 \ln \frac{2 E}{\mu}+4 \operatorname{Li}_{2}(1-x)+\frac{\pi^{2}}{6}-\frac{2}{u}\left[\frac{\ln x}{(1-x)^{2}}-\frac{\ln x \bar{u}}{(1-x \bar{u})^{2}}\right.\right. \\
& \left.\left.+\frac{\ln x}{1-x}-\frac{\ln x \bar{u}}{1-x \bar{u}}-2 \ln \bar{u}\right]+\frac{6 \ln x}{1-x}-\frac{2 x}{(1-x)(1-x \bar{u})}+2\right\} \\
+ & \left(C_{F}-\frac{C_{A}}{2}\right) \frac{\alpha_{s}}{4 \pi}\left\{\frac{4}{\bar{u}}\left[2\left(\ln \frac{2 E}{\mu}-1\right) \ln u+\ln ^{2} u-\operatorname{Li}_{2}(1-x)+\operatorname{Li}_{2}(1-x u)\right]\right. \\
& \left.+\frac{4}{u}\left[\frac{\ln x}{1-x}-\frac{\ln x \bar{u}}{1-x \bar{u}}-\ln \bar{u}\right]\right\}
\end{aligned}
$$




$$
\begin{aligned}
& C_{V 3}^{B^{\prime}}=1-x+\frac{C_{F} \alpha_{s}}{4 \pi}\left\{(1-x)\left[-2 \ln ^{2} \frac{2 E}{\mu}+\ln \frac{2 E}{\mu}-2 \operatorname{Li}_{2}(1-x)-\frac{\pi^{2}}{12}\right]\right. \\
& -\left[\frac{1}{(1-x \bar{u})^{2}}+3-2 x\right] \frac{\ln \bar{u}}{u}+\left[\frac{x}{(1-x \bar{u})^{2}}+\frac{x}{(1-x)(1-x \bar{u})}-3\right] \frac{\ln x}{1-x} \\
& \left.+\frac{x}{(1-x)(1-x \bar{u})}-2+x\right\} \\
& +\left(C_{F}-\frac{C_{A}}{2}\right) \frac{\alpha_{s}}{4 \pi}\left\{\frac{2}{x u \bar{u}}\left[\operatorname{Li}_{2}(1-x)-\mathrm{Li}_{2}(1-x u)-\mathrm{Li}_{2}(1-x \bar{u})+\frac{\pi^{2}}{6}\right]\right. \\
& +\frac{2(2-x) \ln u}{\bar{u}}+2\left[\frac{1}{1-x \bar{u}}+2-x\right] \frac{\ln \bar{u}}{u}-\frac{2 x \ln x}{(1-x)(1-x \bar{u})} \\
& \left.-\frac{2(1-x)}{\bar{u}}\left[\left(2 \ln \frac{2 E}{\mu}-1\right) \ln u+\ln ^{2} u-\operatorname{Li}_{2}(1-x)+\operatorname{Li}_{2}(1-x u)\right]\right\}, \\
& C_{V 4}^{B^{\prime}}=\frac{C_{F} \alpha_{s}}{4 \pi} \frac{x}{1-x \bar{u}}\left\{-\left[\frac{1}{1-x \bar{u}}+\frac{1}{u}\right] \ln \bar{u}-\left[\frac{1}{1-x \bar{u}}-\frac{x}{1-x}\right] \ln x-1\right\} \\
& +\left(C_{F}-\frac{C_{A}}{2}\right) \frac{\alpha_{s}}{4 \pi}\left\{-\frac{2(1-x) \ln u}{\bar{u}(1-x u)}-\frac{2(1-x) \ln \bar{u}}{u(1-x \bar{u})}+\frac{2 x(2-x) \ln x}{(1-x u)(1-x \bar{u})}\right. \\
& \left.-\frac{2}{x u \bar{u}}\left[\operatorname{Li}_{2}(1-x)-\operatorname{Li}_{2}(1-x u)-\operatorname{Li}_{2}(1-x \bar{u})+\frac{\pi^{2}}{6}\right]\right\} \text {. }
\end{aligned}
$$

The three coefficients needed for the tensor case are

$$
\begin{gathered}
C_{T 4}^{B^{\prime}=2+} \frac{C_{F} \alpha_{s}}{4 \pi}\left\{-4 \ln ^{2} \frac{2 E}{\mu}+2 \ln \frac{2 E}{\mu}-4 \ln \frac{\mu_{\mathrm{QCD}}}{2 E}-4 \operatorname{Li}_{2}(1-x)-\frac{\pi^{2}}{6}\right. \\
+2\left[\frac{1-x}{(1-x \bar{u})^{2}}-\frac{2+x}{1-x \bar{u}}-3\right] \frac{\ln \bar{u}}{u} \\
\left.-2\left[\frac{x}{(1-x \bar{u})^{2}}-\frac{x(1+x)}{(1-x)(1-x \bar{u})}+5\right] \ln x-\frac{2 x}{1-x \bar{u}}-4\right\} \\
+\left(C_{F}-\frac{C_{A}}{2}\right) \frac{\alpha_{s}}{4 \pi}\left\{-\frac{4}{\bar{u}}\left[\left(2 \ln \frac{2 E}{\mu}-1\right) \ln u+\ln ^{2} u-\operatorname{Li}_{2}(1-x)+\operatorname{Li}_{2}(1-x u)\right]\right. \\
-4\left[\frac{1-x}{1-x \bar{u}}-2\right] \frac{\ln \bar{u}}{u}+\frac{4 x \ln x}{1-x \bar{u}}
\end{gathered}
$$




$$
\begin{aligned}
& \left.-\frac{4}{x u \bar{u}}\left[\operatorname{Li}_{2}(1-x)-\operatorname{Li}_{2}(1-x u)-\mathrm{Li}_{2}(1-x \bar{u})+\frac{\pi^{2}}{6}\right]\right\}, \\
C_{T 6}^{B^{\prime}}=0, & \\
C_{T 7}^{B^{\prime}}=2 x+ & \frac{C_{F} \alpha_{s}}{4 \pi} x\left\{-4 \ln ^{2} \frac{2 E}{\mu}+2 \ln \frac{2 E}{\mu}-4 \ln \frac{\mu_{\mathrm{QCD}}}{2 E}-4 \operatorname{Li}_{2}(1-x)-\frac{\pi^{2}}{6}\right. \\
+ & \left.\frac{4 \bar{u}}{u}\left[\left(2 \ln \frac{2 E}{\mu}-1\right) \ln \bar{u}+\ln ^{2} \bar{u}-\operatorname{Li}_{2}(1-x)+\operatorname{Li}_{2}(1-x \bar{u})\right]-\frac{4}{u} \ln \bar{u}-2 \ln x-2\right\} \\
+ & \left(C_{F}-\frac{C_{A}}{2}\right) \frac{\alpha_{s}}{4 \pi} x\left\{-\frac{4 \bar{u}}{u}\left[\left(2 \ln \frac{2 E}{\mu}-1\right) \ln \bar{u}+\ln ^{2} \bar{u}-\operatorname{Li}_{2}(1-x)+\operatorname{Li}_{2}(1-x \bar{u})\right]\right. \\
& \quad-\frac{4(2-u)}{\bar{u}}\left[\left(2 \ln \frac{2 E}{\mu}-1\right) \ln u+\ln ^{2} u-\operatorname{Li}_{2}(1-x)+\operatorname{Li}_{2}(1-x u)\right] \\
& \left.\quad-\frac{4}{x u \bar{u}}\left[\operatorname{Li}_{2}(1-x)-\operatorname{Li}_{2}(1-x u)\right]+\frac{4}{x u}\left[\operatorname{Li}_{2}(1-x \bar{u})-\frac{\pi^{2}}{6}\right]+4 \ln \bar{u}-4 \ln u-4\right\} .
\end{aligned}
$$

We have checked that our results for all four vector currents, and seven tensor currents (including those not listed here) are in complete agreement with previous results of Beneke, Kiyo and Yang [17] once we translate to their operator basis, using the relations

$$
\begin{aligned}
C_{V 1}^{B^{\prime}} & =C_{V}^{(A 1) 1}+\frac{1}{2} C_{V}^{(A 1) 4}+x C_{V}^{(B 1) 1}+\frac{x}{2} C_{V}^{(B 1) 4}, & C_{V 2}^{B^{\prime}} & =-2 C_{V}^{(A 1) 3}+x C_{V}^{(B 1) 3} \\
C_{V 3}^{B^{\prime}} & =C_{V}^{(A 1) 2}-x C_{V}^{(B 1) 2}, & C_{V 4}^{B^{\prime}} & =\frac{1}{2} C_{V}^{(A 1) 4}+\frac{x}{2} C_{V}^{(B 1) 4}
\end{aligned}
$$

for the vector coefficients, and for the tensor coefficients:

$$
\begin{array}{ll}
C_{T 1}^{B^{\prime}}=-C_{T}^{(A 1) 4}-x C_{T}^{(B 1) 4}, & C_{T 2}^{B^{\prime}}=-4 C_{T}^{(A 1) 2}+C_{T}^{(A 1) 6}-2 x C_{T}^{(B 1) 2}+C_{T}^{(B 1) 6}, \\
C_{T 4}^{B^{\prime}}=2 C_{3}^{(A 1) 3}-2 x C_{T}^{(B 1) 3}, & C_{T 3}^{B^{\prime}}=2 C_{T}^{(A 1) 1}+C_{T}^{(A 1) 5}-2 x C_{T}^{(B 1) 1}+2 x C_{T}^{(B 1) 5}, \\
C_{T 6}^{B^{\prime}}=C_{T}^{(A 1) 6}+x C_{T}^{(B 1) 6}, & C_{T 5}^{B^{\prime}}=C_{T}^{(A 1) 4}-C_{T}^{(A 1) 7}+x C_{T}^{(B 1) 4}-x C_{T}^{(B 1) 7}, \\
C_{T 7}^{B^{\prime}}=C_{T}^{(A 1) 5}+2 x C_{T}^{(B 1) 5} . &
\end{array}
$$

\section{One-loop matching from $\mathrm{SCET}_{\mathrm{I}}$ onto $\mathrm{SCET}_{\mathrm{II}}$}

We now map the $B$-type $\mathrm{SCET}_{\mathrm{I}}$ currents onto $\mathrm{SCET}_{\mathrm{II}}$ four-quark operators, as represented in the right-hand side of Figure 1 . We recall that $\mathrm{SCET}_{\mathrm{II}}$ is the low-energy effective theory encompassing collinear modes with momentum scaling $\left(n \cdot p, \bar{n} \cdot p, p_{\perp}\right) \sim\left(\lambda^{2}, 1, \lambda\right)$, and soft modes with momentum scaling $(\lambda, \lambda, \lambda)$. In general, soft-collinear messenger modes, with 

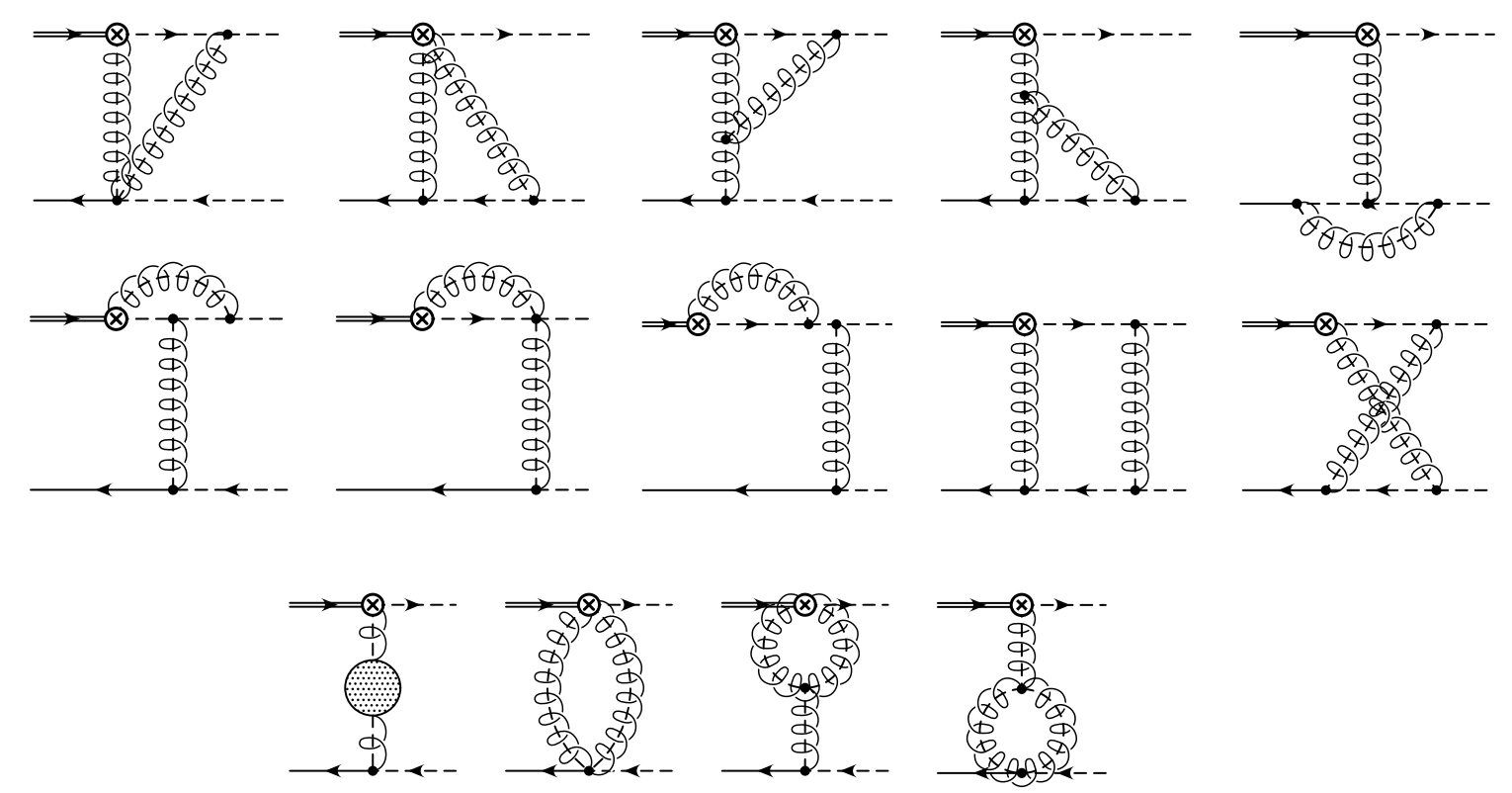

Figure 3: $\mathrm{SCET}_{\mathrm{I}}$ graphs contributing to the matching of the currents $J_{j}^{B}$ (crossed circle) onto the $\mathrm{SCET}_{\mathrm{II}}$ four-quark operators $O_{i}$. Full lines denote soft fields, dashed lines hard-collinear fields. Diagrams with soft gluons or scaleless loops are not shown.

momentum scaling $\left(\lambda^{2}, \lambda, \lambda^{3 / 2}\right)$ are also required, even at leading order, and may communicate between the soft and collinear sectors [22]. However, in the particular case of the color-singlet four-quark operators under consideration, the sum over all such contributions can be shown to vanish; this is equivalent to the statement that the soft and collinear sectors factorize for the corresponding matrix elements $[23,13]$. The resulting factorizable contributions to the form factors take the form of the second term in (1). The function $T_{i}(E, \omega, u)$ appearing in this formula is a product (in general, a convolution) of the $\operatorname{SCET}_{\mathrm{I}}$ coefficients $C_{i}^{B}$ from the first matching step, considered in Section 2, and the Wilson coefficients occurring in the second matching step, called jet-functions. The fact that the heavy quark and the collinear quarks are described by two component spinors restricts the allowed form of possible $\mathrm{SCET}_{\mathrm{II}}$ operators, and leads to relations among the jet-functions [16]. For the operators relevant to $B$-decay form factors, only two jet functions are relevant; these are denoted by $\mathcal{J}_{\|}$and $\mathcal{J}_{\perp}$, and describe decays into pseudoscalar or longitudinally-polarized vector mesons, and into perpendicularly-polarized vector mesons, respectively. For completeness, and to illustrate the effects of evanescent operators, we will present in this section complete one-loop results for all jet functions.

The $B$-type currents in $\mathrm{SCET}_{\mathrm{I}}$ may be written,

$$
J_{\Gamma}(s, r)=\bar{X}_{h c}(s \bar{n}) \mathcal{A}_{h c \perp \mu}(r \bar{n}) \Gamma_{\perp}^{\mu} h(0),
$$

where the Dirac structure $\Gamma_{\perp}^{\mu}$ depends on the specific current under consideration; e.g. $\Gamma_{\perp}^{\mu}=\gamma_{\perp}^{\mu}$ for the scalar current. For the coefficient function of the operator $J_{\Gamma}(s, r)$, we write

$$
C_{\Gamma}(u)=\int d r d s e^{i(u s+\bar{u} r) \bar{n} \cdot P} \tilde{C}_{\Gamma}(s, r)
$$


The four-quark SCET II operators depicted in Figure 1 take the form,

$$
O_{\Gamma, i}^{k l, m n}(s, t)=\bar{X}_{c}^{k}(s \bar{n}) \Gamma_{\bar{\xi} h, i} \mathcal{H}^{l}(0) \bar{Q}_{s}^{m}(t n) \Gamma_{\bar{q} \xi, i} X_{c}^{n}(0) .
$$

The superscripts $k, l, m, n$ are color indices, which will be suppressed in the following. Since we are interested in matrix elements for color-singlet initial and final state hadrons, our primary concern is the color-singlet contribution, proportional to the contraction of (15) with $\delta^{k n} \delta^{m l}$. Here $X_{c}=W_{c}^{\dagger} \xi_{c}$ is the restriction of $X_{h c}$ to collinear momentum modes. $\mathcal{H}=S^{\dagger} h$ and $Q_{s}=S^{\dagger} q_{s}$ are soft fields multiplied by a soft Wilson line $S$ in the $n$-direction. The precise definitions of these fields and the $\mathrm{SCET}_{\mathrm{II}}$ effective Lagrangian is given in [22].

Unlike in the first matching step, from QCD onto $\mathrm{SCET}_{\mathrm{I}}$, the matching of $\mathrm{SCET}_{\mathrm{I}}$ onto $\mathrm{SCET}_{\mathrm{II}}$ generates an infinite tower of $\mathrm{SCET}_{\mathrm{II}}$ operators. From the Feynman rules of $\mathrm{SCET}_{\mathrm{I}}$ it follows that $\Gamma_{\bar{q} \xi}$ must take the form

$$
\Gamma_{\bar{q} \xi}=\gamma_{\perp \mu}, \gamma_{\perp \mu_{1} \mu_{2} \mu_{3}}, \gamma_{\perp \mu_{1} \mu_{2} \mu_{3} \mu_{4} \mu_{5}}, \ldots
$$

We denote totally antisymmetrized combinations of Dirac matrices by $\gamma_{\perp}^{\mu_{1} \ldots \mu_{n}}=\gamma_{\perp}^{\left[\mu_{1}\right.} \ldots \gamma_{\perp}^{\left.\mu_{n}\right]}$. A complete basis is obtained by considering the most general $\Gamma_{\bar{\xi} h}$ with the appropriate number of Lorentz indices, constructed from $\Gamma_{\perp}^{\mu}$ and $\gamma_{\perp}^{\mu}$ (recall that we choose the heavy-quark velocity such that $\left.v_{\perp}^{\mu}=0\right)$. At one-loop order, three structures are possible:

$$
\Gamma_{\bar{\xi} h, i} \otimes \Gamma_{\bar{q} \xi, i}=\left\{\begin{aligned}
\Gamma_{\perp}^{\mu} \otimes \gamma_{\perp \mu}, & i & =1, \\
\gamma_{\perp}^{\mu} \gamma_{\perp \rho} \Gamma_{\perp}^{\rho} \otimes \gamma_{\perp \mu}, & i & =2, \\
\gamma_{\perp}^{\mu \nu} \Gamma_{\perp}^{\rho} \otimes \gamma_{\perp \mu \nu \rho}, & i & =3 .
\end{aligned}\right.
$$

Operators for which $\Gamma_{\bar{q} \xi}$ involves antisymmetric products of five and more Dirac matrices appear only at two and more loops. In four dimensions, antisymmetric products of more than two perpendicular Dirac matrices vanish. Operators such as $O_{\Gamma, 3}(s, t)$ containing these Dirac structures appear at one-loop and beyond in dimensional regularization, and are called evanescent $[18,19,20]$. The choice of evanescent operators is not unique, and affects the coefficients of the physical operators. We will discuss this issue, and the related subject of renormalization in the presence of evanescent operators in Section 4. In this section we present results for the bare matching coefficients.

We define the Fourier transform of the coefficient of the $\operatorname{SCET}_{\mathrm{II}}$ operators $O_{\Gamma, i}(s, t)$ as

$$
D_{\Gamma, i}(\omega, u)=\int d s d t e^{-i \omega n \cdot v t} e^{i u s \bar{n} \cdot P} \tilde{D}_{\Gamma, i}(s, t),
$$

where $\tilde{D}_{\Gamma, i}(s, t)$ is the position-space coefficient. The jet functions relating bare coefficients are defined via

$$
D_{\Gamma, i}^{\mathrm{bare}}(\omega, u)=\frac{1}{2 E \omega} \int_{0}^{1} d v \mathcal{J}_{i}\left(u, v, g^{2}(2 E \omega)^{-\epsilon}\right) C_{\Gamma}^{\mathrm{bare}}(v),
$$

which we expand as

$$
\mathcal{J}_{i}\left(u, v, g^{2}(2 E \omega)^{-\epsilon}\right)=\frac{g^{2}}{2 E \bar{u}}\left(-\delta_{i 1} \delta(u-v)(\mathcal{S}+\mathcal{O})\right.
$$




$$
\left.+\frac{g^{2}}{(4 \pi)^{2-\epsilon}} \Gamma(1+\epsilon)(2 E \omega)^{-\epsilon} J_{i}^{(1)}(u, v)+\ldots\right) .
$$

Here $g$ is the bare coupling; the dependence of $\mathcal{J}_{i}$ on $g, E$ and $\omega$ is determined by dimensional analysis and invariance under rescaling of the light-cone vector $n$. The symbols $\mathcal{S}$ and $\mathcal{O}$ represent the color-singlet and color-octet configurations of four-quark operators. Restoring the color indices which are to be contracted with the operators in (15), we define:

$$
\begin{aligned}
\mathcal{S}^{k l, m n} & =\frac{C_{F}}{N} \delta^{k n} \delta^{m l} \\
\mathcal{O}^{k l, m n} & =-\frac{1}{N}\left(T_{A}\right)^{k n}\left(T_{A}\right)^{m l},
\end{aligned}
$$

where for convenience we include the tree-level factors in the definitions of $\mathcal{S}$ and $\mathcal{O}$.

The one-loop jet-functions for the three operators in (17) are obtained from the $\mathrm{SCET}_{\mathrm{I}}$ diagrams shown in Figure 3. Diagrams involving soft modes need not be considered, since these momentum regions are reproduced exactly by the corresponding $\mathrm{SCET}_{\mathrm{II}}$ contributions, and hence cancel in the matching. We proceed by evaluating the loop diagrams for an arbitrary coefficient function $C_{\Gamma}$ in (14). To perform the integration over the $d$-dimensional loop momentum $k$, we first integrate over the $n \cdot k$ component using the method of residues, and then perform the $k_{\perp}$ integral for the $d-2$ perpendicular dimensions. The remaining integration over $\bar{n} \cdot k$ takes the form of equation (19), from which we read off the contributions to the jet-functions. In this way, we find

$$
\begin{aligned}
J_{1}^{(1)}(u, v)= & {\left[\frac{\theta(u-v)}{u-v}(u-v)^{-\epsilon}+\frac{\theta(v-u)}{v-u}(v-u)^{-\epsilon}\right]_{+}\left[\left(C_{F}-\frac{C_{A}}{2}\right) \mathcal{S}+\left(C_{F}-C_{A}\right) \mathcal{O}\right] \frac{2}{\epsilon} } \\
& +\theta(u-v)(u-v)^{-\epsilon}\left[\left(C_{F}-\frac{C_{A}}{2}\right) \mathcal{S}+\left(C_{F}-C_{A}\right) \mathcal{O}\right] \\
& \times \frac{2}{u \bar{v}}\left[-\frac{1}{\epsilon}+\frac{v \bar{u}}{u-v} \ln \frac{u}{v}-\frac{1}{2}(1+v)\right] \\
& +\theta(v-u)(v-u)^{-\epsilon}\left\{\left[C_{F}(\mathcal{S}+\mathcal{O})-\frac{C_{A}}{2}\left(\frac{\bar{v}}{\bar{u}} \mathcal{S}+\left[1+\frac{\bar{v}}{\bar{u}}\right] \mathcal{O}\right)\right]\right. \\
& \left.\times \frac{2}{u \bar{v}}\left[\frac{u \bar{v}}{v-u} \ln \frac{\bar{u}}{\bar{v}}-\frac{1}{2}(1+u)\right]+\frac{C_{A}}{2}(\mathcal{S}+\mathcal{O}) \frac{2}{\bar{u}} \frac{1}{\epsilon}\right\} \\
& +[v \bar{v}]^{-\epsilon} \frac{1}{u \bar{v}}\left\{C_{F}(\mathcal{S}+\mathcal{O})[(1+u) v-\bar{v}]-\frac{C_{A}}{2}[v(1+u) \mathcal{O}-\bar{v} \mathcal{S}]\right\} \\
& +\theta(1-u-v)\left[\frac{v(1-u-v)}{\bar{u}}\right]^{-\epsilon}\left(C_{F}-\frac{C_{A}}{2}\right)(\mathcal{S}+\mathcal{O}) \frac{2 v}{\bar{u} \bar{v}}\left(-\frac{1}{\epsilon}+\frac{\bar{v}}{u v}-\frac{1}{v}\right) \\
& +\delta(u-v) u^{-\epsilon}\left[\left(C_{F}-\frac{C_{A}}{2}\right) \mathcal{S}+\left(C_{F}-C_{A}\right) \mathcal{O}\right]\left(-\frac{2}{\epsilon^{2}}\right)
\end{aligned}
$$




$$
\begin{aligned}
& +\delta(u-v) \bar{u}^{-\epsilon}\left\{-\frac{C_{A}}{2} \mathcal{S}\left(\frac{2}{\epsilon^{2}}\right)+(\mathcal{S}+\mathcal{O})\left[\left(\frac{4}{3} T_{F} n_{f}+3 C_{F}-\frac{11}{3} C_{A}\right)\left(\frac{1}{\epsilon}\right)\right.\right. \\
& \left.\left.+\frac{20}{9} T_{F} n_{f}+8 C_{F}-\frac{76}{9} C_{A}-\left(C_{F}-C_{A}\right) \frac{\pi^{2}}{3}\right]\right\} \\
& J_{2}^{(1)}(u, v)=\theta(u-v)\left[\frac{(u-v) v}{u}\right]^{-\epsilon}\left[\left(C_{F}-\frac{C_{A}}{2}\right) \mathcal{S}+\left(C_{F}-C_{A}\right) \mathcal{O}\right] \frac{\bar{u}}{u \bar{v}}\left(\frac{1}{\epsilon}+1\right) \\
& +\theta(v-u)\left[\frac{(v-u) \bar{v}}{\bar{u}}\right]^{-\epsilon}\left[C_{F}(\mathcal{S}+\mathcal{O})-\frac{C_{A}}{2}\left(\frac{\bar{v}}{\bar{u}} \mathcal{S}+\left(1+\frac{\bar{v}}{\bar{u}}\right) \mathcal{O}\right)\right] \frac{1}{u}\left(\frac{1}{\epsilon}+1\right) \\
& +[v \bar{v}]^{-\epsilon}\left[C_{F}(\mathcal{S}+\mathcal{O}) \frac{v \bar{u}}{u}\left(-\frac{1}{\epsilon}+\frac{\bar{v}}{v \bar{u}}\right)+\frac{C_{A}}{2}\left(-\frac{1}{u} \mathcal{S}+\mathcal{O} \frac{\bar{u}}{u}\left[\frac{1}{\epsilon}-\frac{u}{\bar{u}}\right]\right)\right] \\
& +\theta(1-u-v)\left[\frac{(1-u-v) v}{\bar{u}}\right]^{-\epsilon}\left(C_{F}-\frac{C_{A}}{2}\right)(\mathcal{S}+\mathcal{O}) \\
& \times \frac{1}{u \bar{u} \bar{v}}\left[\left(u v-[1-u-v]^{2}\right) \frac{1}{\epsilon}-(1-u-v)(2-u-v)\right], \\
& J_{3}^{(1)}(u, v)=\theta(u-v)\left[\frac{(u-v) v}{u}\right]^{-\epsilon}\left[\left(C_{F}-\frac{C_{A}}{2}\right) \mathcal{S}+\left(C_{F}-C_{A}\right) \mathcal{O}\right] \frac{1}{2 u}\left(\frac{1}{\epsilon}+1\right) \\
& +\theta(v-u)\left[\frac{(v-u) \bar{v}}{\bar{u}}\right]^{-\epsilon}\left[C_{F}(\mathcal{S}+\mathcal{O})-\frac{C_{A}}{2}\left(\frac{\bar{v}}{\bar{u}} \mathcal{S}+\left(1+\frac{\bar{v}}{\bar{u}}\right) \mathcal{O}\right)\right] \frac{\bar{u}}{2 u \bar{v}}\left(\frac{1}{\epsilon}+1\right) \\
& +[v \bar{v}]^{-\epsilon}\left[C_{F}(\mathcal{S}+\mathcal{O}) \frac{1-u v}{2 u \bar{v}}\left(-\frac{1}{\epsilon}-1\right)\right. \\
& \left.-\frac{C_{A}}{2}\left(\frac{1}{2 u} \mathcal{S}+\left[\frac{1}{2 u}+\frac{1-u v}{2 u \bar{v}}\right] \mathcal{O}\right)\left(-\frac{1}{\epsilon}-1\right)\right] .
\end{aligned}
$$

The plus distribution appearing in $J_{1}^{(1)}$ is defined for symmetric functions $g(u, v)$ to act on test functions $f(v)$ as

$$
\int d v[g(u, v)]_{+} f(v)=\int d v g(u, v)[f(v)-f(u)] .
$$

We now return to the particular cases of the scalar, vector and tensor current operators and extract their jet-functions from the general expressions above. Through one-loop order, one physical and one evanescent four-quark operator are sufficient for the scalar case:

$$
O_{S 1}=\gamma_{\perp}^{\alpha} \otimes \gamma_{\perp \alpha}, \quad O_{S 2}=\gamma_{\perp}^{\alpha \beta \gamma} \otimes \gamma_{\perp \alpha \beta \gamma}
$$


To the same order, there are four physical and four evanescent vector operators

$$
\begin{array}{llll}
O_{V 1}^{\mu}=\frac{n^{\mu}}{n \cdot v} O_{S 1}, & O_{V 2}^{\mu}=v^{\mu} O_{S 1}, & O_{V 3}^{\mu}=\gamma_{\perp}^{\mu} \gamma_{\perp}^{\alpha} \otimes \gamma_{\perp \alpha}, & O_{V 4}^{\mu}=\gamma_{\perp}^{\alpha} \gamma_{\perp}^{\mu} \otimes \gamma_{\perp \alpha}, \\
O_{V 5}^{\mu}=\frac{n^{\mu}}{n \cdot v} O_{S 2}, & O_{V 6}^{\mu}=v^{\mu} O_{S 2}, & O_{V 7}^{\mu}=\gamma_{\perp}^{\alpha \beta} \gamma_{\perp}^{\mu} \gamma_{\perp}^{\gamma} \otimes \gamma_{\perp \alpha \beta \gamma}, & O_{V 8}^{\mu}=\gamma_{\perp}^{\alpha \beta \gamma} \gamma_{\perp}^{\mu} \otimes \gamma_{\perp \alpha \beta \gamma},
\end{array}
$$

and a grand total of fourteen tensor operators

$$
\begin{aligned}
& O_{T 1}^{\mu \nu}=\frac{n^{[\mu} v^{\nu]}}{n \cdot v} O_{S 1}, \quad O_{T 2}^{\mu \nu}=\frac{1}{n \cdot v} n^{[\mu} O_{V 3}^{\nu]}, \quad O_{T 3}^{\mu \nu}=v^{[\mu} O_{V 3}^{\nu]}, \quad O_{T 4}^{\mu \nu}=\frac{1}{n \cdot v} n^{[\mu} O_{V 4}^{\nu]}, \\
& O_{T 5}^{\mu \nu}=v^{[\mu} O_{V 4}^{\nu]}, \quad O_{T 6}^{\mu \nu}=\gamma_{\perp}^{\alpha} \gamma_{\perp}^{\mu \nu} \otimes \gamma_{\perp \alpha}, \quad O_{T 7}^{\mu \nu}=\gamma_{\perp}^{\alpha \mu \nu} \otimes \gamma_{\perp \alpha}, \quad O_{T 8}^{\mu \nu}=\frac{n^{[\mu} v^{\nu]}}{n \cdot v} O_{S 2}, \\
& O_{T 9}^{\mu \nu}=\frac{1}{n \cdot v} n^{[\mu} O_{V 7}^{\nu]}, \quad O_{T 10}^{\mu \nu}=v^{[\mu} O_{V 7}^{\nu]}, \quad O_{T 11}^{\mu \nu}=\frac{1}{n \cdot v} n^{[\mu} O_{V 8}^{\nu]}, \quad O_{T 12}^{\mu \nu}=v^{[\mu} O_{V 8}^{\nu]}, \\
& O_{T 13}^{\mu \nu}=\gamma_{\perp}^{\alpha \beta \gamma} \gamma_{\perp}^{\mu \nu} \otimes \gamma_{\perp \alpha \beta \gamma}, \quad O_{T 14}^{\mu \nu}=\gamma_{\perp}^{\alpha \beta} \gamma_{\perp}^{\gamma \mu \nu} \otimes \gamma_{\perp \alpha \beta \gamma},
\end{aligned}
$$

of which the last eight are evanescent; in particular, $O_{T 7}$, though appearing already at treelevel, is evanescent. ${ }^{2}$ As is evident from the above equations many of these operators are directly related to each other, e.g. $O_{V 1}$ to $O_{S 1}$, etc., and the matching onto these operators involves the same jet-functions. To obtain the jet-function in a particular case, we first rewrite the $\mathrm{SCET}_{\mathrm{I}}$ current in the general form (13), and then evaluate the three corresponding operators of (17). Matching to the basis of four-quark operators defined in (26), (27) and (28) yields the jet-functions in terms of the functions $\mathcal{J}_{i}$ defined in (19). The independent matching relations are obtained from those operators with no factors of $v^{\mu}$ or $n^{\mu}$, and with zero, one or two perpendicular Lorentz indices:

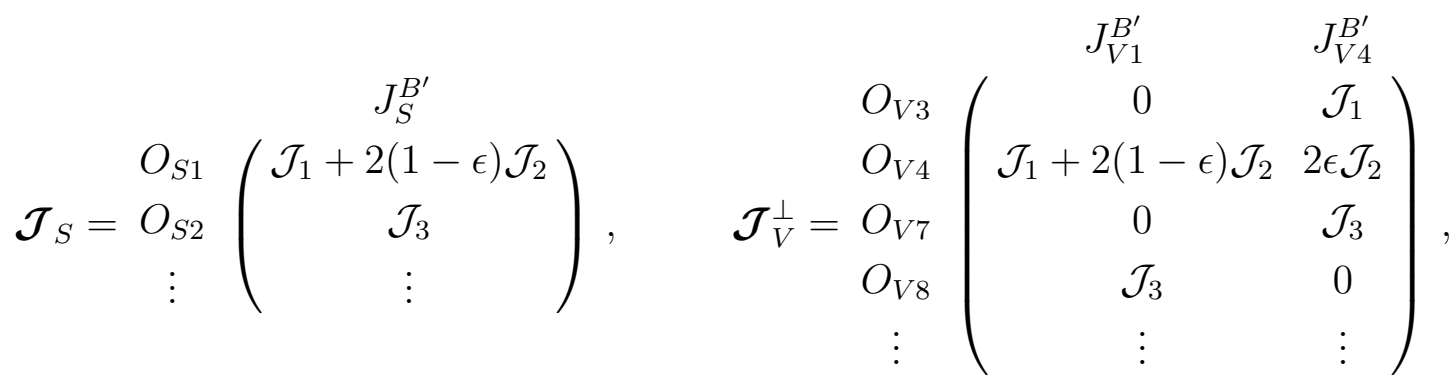

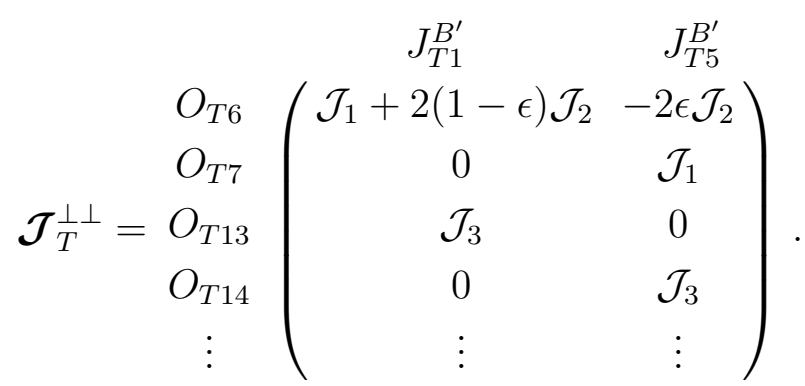

\footnotetext{
${ }^{2}$ Although three independent structures are possible for strings of five $\gamma_{\perp}$ matrices with the appropriate antisymmetrizations, only $O_{T 13}$ and $O_{T 14}$ arise in the matching.
} 
Since they are related to the operators above simply by overall factors of $v^{\mu}$ and $n^{\mu}$, the remaining matching relations onto $O_{V 1,2,5,6}$ and onto $O_{T 1, \ldots, 5,8, \ldots, 12}$ may be obtained from (29), using (26), (27) and (28), and the analogous relations for the SCET I currents in (7) and (8).

From (29), we note that for a particular $\mathrm{SCET}_{\mathrm{I}}$ current $J_{\Gamma}$ in (13), the index contractions in the resulting $\mathrm{SCET}_{\mathrm{II}}$ operators $O_{\Gamma, 2}$ and $O_{\Gamma, 3}$ in (17) lead to $\epsilon$-dependent prefactors, which need to be expanded around $d=4$ before renormalization in order to arrive at $\overline{\mathrm{MS}}$ subtracted jet-functions. Before performing this renormalization, we first consider carefully the renormalization scheme dependence implicit in our above choice of evanescent operator basis.

\section{Evanescent Operators in SCET}

We have seen in the previous section that in dimensional regularization, an infinite tower of evanescent operators is generated upon matching $\mathrm{SCET}_{\mathrm{I}}$ onto $\mathrm{SCET}_{\mathrm{II}}$. These operators can mix into the physical basis, and so their effects must be included for a consistent analysis. To ensure that these operators do not introduce additional nonperturbative parameters into the theory, it is important to show that a renormalization scheme can be chosen such that their renormalized matrix elements vanish. Here we show that this is in fact the case. A related issue concerns the choice of evanescent operator basis. As we will discuss, the renormalized matrix elements of physical operators depend on this basis choice, so that choosing a particular basis of evanescent operators induces a renormalization scheme dependence in the physical operators. For the four-quark SCET II operators defining the hard-scattering part of the form factors, we choose the basis such that the resulting renormalization scheme corresponds to the $\overline{\mathrm{MS}}$ scheme after Fierz transformation. In Section 4.1 we present a general discussion of evanescent operators in SCET, while in Section 4.2 we apply the general formalism to the operators appearing in the form factor analysis.

\subsection{Evanescent operators and the Sudakov problem}

Evanescent operators first appeared in the case of local four-fermion interactions [18]. At one-loop order, it is easy to see that the Green functions of bare evanescent operators are finite and local, and hence can be made to vanish by the addition of a suitable counterterm. The finiteness of the Green functions results from the $1 / \epsilon$ pole from the momentum integral of the corresponding Feynman diagrams being cancelled by a factor $\epsilon$ in the Dirac algebra; this latter factor of $\epsilon$ always appears when evanescent operators "cross the threshold" and mix into physical operators, reflecting the fact that evanescents, and hence any contractions involving them, vanish in $d=4$. The locality of these particular Green functions is a consequence of the locality of the coefficient of the $1 / \epsilon$ pole of one-loop integrals. A renormalization scheme in which matrix elements of evanescent operators vanish to all orders in perturbation theory may then be established by induction [19]. In the present situation, where Green functions of the (nonlocal) SCET current operators contain $1 / \epsilon^{2}$ double poles already at one-loop order, one may ask if an analogous renormalization scheme for evanescent operators exists. We will show that this is indeed the case, both for $\mathrm{SCET}_{\mathrm{I}}$ and $\mathrm{SCET}_{\mathrm{II}}$. In practical applications where 
we will ultimately match onto $\mathrm{SCET}_{\mathrm{II}}$, we may simply use the conventional $\overline{\mathrm{MS}}$ scheme also for evanescent $\mathrm{SCET}_{\mathrm{I}}$ operators and perform finite subtractions only in the final low energy theory. This is the procedure adopted in Section 4.2.

Let us consider first the simpler case of the evanescent SCET $\mathrm{I}$ current operator, $J_{T 5}^{B^{\prime}}$, denoted by $J_{T 5}$ in the following. Under renormalization, this operator mixes with $J_{T 1}^{B^{\prime}}$, denoted by $J_{T 1} \cdot{ }^{3}$ We proceed to isolate the linear combination of $J_{T 1}$ and $J_{T 5}$ which corresponds to the physical renormalized operator. It will be convenient to define the following notation for these operators, considered as bare quantities,

$$
\begin{aligned}
& \partial_{1}(s, r)=J_{T 5}^{B^{\prime} \mu \nu}(s, r)=\bar{X}(s \bar{n}) \mathcal{A}_{\perp \alpha}(r \bar{n}) \gamma_{\perp}^{\alpha \mu \nu} h(0), \\
& \partial_{2}(s, r)=\bar{X}(s \bar{n}) \mathcal{A}_{\perp}(r \bar{n}) \gamma_{\perp \alpha} \gamma_{\perp}^{\alpha \mu \nu} h(0)=-2 \epsilon \bar{X}(s \bar{n}) \mathcal{A}_{\perp}(r \bar{n}) \gamma_{\perp}^{\mu \nu} h(0)=-2 \epsilon J_{T 1}^{B^{\prime} \mu \nu}(s, r) .
\end{aligned}
$$

From the Feynman rules of $\mathrm{SCET}_{\mathrm{I}}$ and the projection properties of the spinor field $\mathcal{X}$, it follows that the operators $\mathcal{J}_{1}$ and $\mathcal{J}_{2}$ close under renormalization [16]. Defining renormalized operators via $\mathcal{J}_{i}^{\text {ren }}=\sum_{j} Z_{i j} \mathcal{J}_{j}^{\text {bare }}$, the anomalous dimension matrix takes the form

$$
\boldsymbol{Z}=\left(\begin{array}{cc}
Z_{11} & Z_{12} \\
0 & Z_{22}
\end{array}\right)
$$

where, in the $\overline{\mathrm{MS}}$ scheme, it is understood that only pole terms in the dimensional regulator $\epsilon=2-d / 2$ are kept in $\boldsymbol{Z}-\mathbf{1}^{4}$ If we now consider a Green function with an insertion of the renormalized operator, $\mathcal{J}_{1}^{\text {ren }}$, the result will be the sum of two terms, the first proportional to the tree-level matrix element of $\mathcal{J}_{1}$, and the second proportional to the tree-level matrix element of $\mathcal{J}_{2}$. The renormalization constants of $\boldsymbol{Z}$ ensure that the coefficients for both terms are finite. But $\mathcal{J}_{1}$ is evanescent, and $\mathcal{J}_{2}$ contains an explicit factor of $\epsilon$, so their tree-level matrix elements vanish at $d=4$; since the coefficients have been made finite by renormalization, the final result also vanishes in $d=4$. Returning to our original operators, we define

$$
\left(\begin{array}{c}
J_{T 5}^{\text {ren }} \\
J_{T 1}^{\text {ren }}
\end{array}\right) \equiv\left(\begin{array}{c}
\mathcal{J}_{1}^{\text {ren }} \\
-\frac{1}{2 \epsilon} \mathcal{g}_{2}^{\text {ren }}
\end{array}\right)=\left(\begin{array}{cc}
Z_{11}-2 \epsilon Z_{12} \\
0 & Z_{22}
\end{array}\right)\left(\begin{array}{c}
J_{T 5}^{\text {bare }} \\
J_{T 1}^{\text {bare }}
\end{array}\right) .
$$

$J_{T 1}^{\mathrm{ren}}$ and $J_{T 5}^{\mathrm{ren}}$ are equal to their bare counterparts at tree level, and have finite Green functions to all orders in the loop expansion, so that (32) defines a valid renormalization scheme. Clearly $J_{T 5}^{\text {ren }}$, being equal to $\mathcal{J}_{1}^{\text {ren}}$, has vanishing matrix elements in this scheme. We may note that the renormalization of the new basis involves a finite subtraction from the term $\epsilon Z_{12}$, even if the original matrix $\boldsymbol{Z}$ in (31) is defined in the $\overline{\mathrm{MS}}$ scheme.

None of this argument depended on the order in the loop expansion, or on the choice of renormalization scheme used to renormalize $\mathcal{J}_{1}$ and $\mathcal{J}_{2}$. Also, since the renormalized matrix element of $J_{T 5}$ vanishes regardless of renormalization scale, we find that

$$
0=\frac{d}{d \ln \mu}\left\langle J_{T 5}\right\rangle=-\gamma_{T 5, T 5}\left\langle J_{T 5}\right\rangle-\gamma_{T 5, T 1}\left\langle J_{T 1}\right\rangle=-\gamma_{T 5, T 1}\left\langle J_{T 1}\right\rangle
$$

\footnotetext{
${ }^{3}$ This mixing only begins at two-loop order.

${ }^{4} Z_{22}$ is given by the pole part of $Z_{11}+2(1-\epsilon) Z_{12}[16]$; this fact will not be relevant to our analysis.
} 


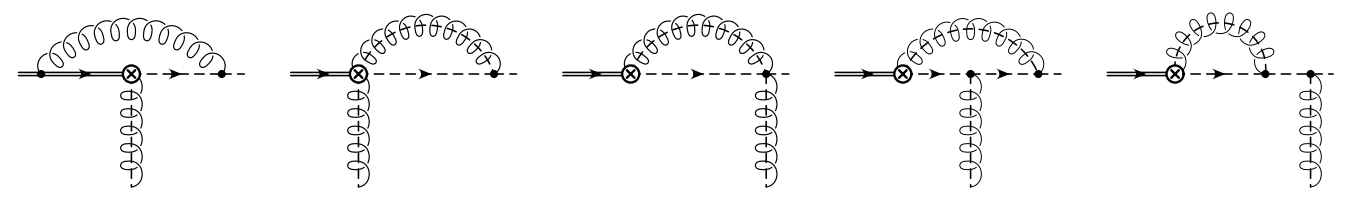

Figure 4: Abelian contribution to the anomalous dimension of the $B$-type $\mathrm{SCET}_{\mathrm{I}}$ current operators. Full lines denote soft fields, dashed lines hard-collinear fields.

where the last equality follows from the vanishing of $\left\langle J_{T 5}\right\rangle$. Since the matrix element of the physical operator is nonzero, it follows that $\gamma_{T 5, T 1}=0$. The evolution equation for the coefficient function of the physical operator is therefore simply

$$
\frac{d}{d \ln \mu} C_{T 1}=\gamma_{T 1, T 1} C_{T 1} .
$$

In particular, the evolution equation is independent of $C_{T 5}$, and the coefficient of the renormalized evanescent operator is irrelevant in the calculation of physical processes.

The simplicity of these arguments hides some interesting implications. For instance, the vanishing of $\gamma_{T 5, T 1}$ implies that at one-loop order $\epsilon Z_{12}$ is finite, or that $Z_{12}$ has at most a single pole at $\epsilon=0$. The one-loop $1 / \epsilon^{2}$ double poles must occur only in the diagonal elements of $\boldsymbol{Z}$, which do not mix the physical and evanescent operators. To illustrate how this works, we recall that the anomalous dimensions of the $B$-type $\mathrm{SCET}_{\mathrm{I}}$ current operators may be extracted from the UV divergences of 3-point Green functions. For simplicity we consider the abelian case, for which the relevant diagrams are shown in Figure 4. The soft contribution is represented by the first diagram, while the remaining diagrams give the hard-collinear contribution. Regulating infrared divergences by evaluating the Green function at an off-shell value of the hard-collinear quark momentum $p$, we find

$$
\begin{aligned}
\text { soft } & \propto\left(\frac{-p^{2}}{2 E \mu}\right)^{-2 \epsilon}\left(-\frac{1}{\epsilon^{2}}+\cdots\right), \\
\text { hard-collinear } & \propto\left(\frac{-p^{2}}{\mu^{2}}\right)^{-\epsilon}\left(\frac{2}{\epsilon^{2}}+\cdots\right) .
\end{aligned}
$$

By general renormalization arguments [24], the coefficient of the $1 / \epsilon$ pole in the one-loop 3-point QCD Green function, which the effective theory is representing, is local and hence independent of $p^{2}$. In fact, in $\mathrm{SCET}_{\mathrm{I}}$, the QCD result is reproduced by including the hard contribution, accounted for by the Wilson coefficient, in addition to the hard-collinear and soft contributions. This hard contribution is independent of $p^{2}$, but may depend on the scales $m_{b}$ and $E$, so that the $\mathrm{SCET}_{\mathrm{I}}$ divergences may be nonlocal over distances $\sim 1 / E$. This is exactly the structure obtained after taking the sum of the two contributions in (35):

$$
\text { soft }+ \text { hard-collinear } \propto \frac{1}{\epsilon^{2}}-\frac{2}{\epsilon} \ln \frac{2 E}{\mu}+\cdots,
$$

where the remaining divergences are local at the level of $\mathrm{SCET}_{\mathrm{I}}$, i.e. independent of $p^{2}$. Since the Dirac structure of the original current is unaffected by the soft gluon exchange, the $1 / \epsilon^{2}$ term in (36) contributes only to the diagonal terms in $\boldsymbol{Z}$. 
We may present similar arguments to show that the renormalized matrix elements of the evanescent $\mathrm{SCET}_{\mathrm{II}}$ operators of the form (15) vanish in $d=4$. Since the heavy quark couples at leading power only through a spin-independent $v \cdot A_{s}$ interaction, no new Dirac structures can be induced by the soft sector. Similarly, the soft-collinear modes couple to collinear modes only through $n \cdot A_{s c}$, and to soft modes only through $\bar{n} \cdot A_{s c}$. All evanescent operators therefore arise from the collinear sector, and we will only write the collinear part of the fourquark operators in our discussion. For simplicity, we restrict attention to the color-singlet case, ${ }^{5}$ and study the operators,

$$
\left[O_{1}\right]_{a b}=\left[\bar{X} \bar{\Gamma}_{\perp}\right]_{a}\left[\Gamma_{\perp} \mathcal{X}\right]_{b}
$$

with spinor indices $a, b$. Here $\bar{\Gamma}_{\perp}, \Gamma_{\perp}$, represent as-yet unspecified Dirac structures $\left(\bar{\Gamma}_{\perp}\right.$ is not related to $\Gamma_{\perp}$ ). It follows from the Feynman rules of $\operatorname{SCET}_{\mathrm{II}}$ that $O_{1}$ mixes with the infinite tower of operators,

$$
\left[O_{n}\right]_{a b}=\left[\bar{X} \gamma_{\perp}^{\nu_{1}} \cdots \gamma_{\perp}^{\nu_{2(n-1)}} \bar{\Gamma}_{\perp}\right]_{a}\left[\Gamma_{\perp} \gamma_{\perp \nu_{2(n-1)}} \cdots \gamma_{\perp \nu_{1}} x\right]_{b}
$$

with $O_{n}$ appearing at $n$-th order in perturbation theory. Here the indices $\nu_{i}$ are not antisymmetrized. By general renormalization arguments [24], renormalization constants $Z_{n m}$ may be chosen so that with $O_{n}^{\text {ren }}=\sum_{m} Z_{n m} O_{m}^{\text {bare }}$, Green functions of the renormalized operators, $O_{n}^{\text {ren }}$, are finite.

In particular, in the representation of the scalar QCD current, we may consider the first evanescent operator, $O_{S 2}$ in (26), for which we take $\bar{\Gamma}_{\perp} \otimes \Gamma_{\perp}=\gamma_{\perp}^{\mu \rho \sigma} \otimes \gamma_{\perp \mu \rho \sigma}$. If we also introduce the operator appearing at tree-level,

$$
\left[O_{0}\right]_{a b}=\left[\bar{X} \gamma_{\perp}^{\mu}\right]_{a}\left[\gamma_{\perp \mu} X\right]_{b}
$$

then the renormalization matrix takes the form, ${ }^{6}$

$$
\left(\begin{array}{c}
O_{0}^{\text {ren }} \\
O_{1}^{\text {ren }} \\
O_{2}^{\text {ren }} \\
\vdots
\end{array}\right)=\left(\begin{array}{cccc}
Z_{00} & Z_{01} & Z_{02} & \cdots \\
0 & Z_{11} & Z_{12} & \cdots \\
0 & Z_{21} & Z_{22} & \cdots \\
\vdots & \vdots & \vdots & \ddots
\end{array}\right)\left(\begin{array}{c}
O_{0}^{\text {bare }} \\
O_{1}^{\text {bare }} \\
O_{2}^{\text {bare }} \\
\vdots
\end{array}\right) .
$$

The reasoning now proceeds as in the previous case. The operators $O_{n}$ for $n \geq 1$ close under renormalization, and after renormalization, a Green function involving the insertion of one of these operators is a linear combination of finite coefficients times tree-level matrix elements. Since the tree-level matrix elements vanish in $d=4$, the renormalized Green functions of the evanescent operators $O_{n}^{\text {ren }}, n \geq 1$, also vanish. Again, since this is true for any renormalization

\footnotetext{
${ }^{5}$ For the color-singlet case, the soft-collinear modes decouple entirely [23], so that the collinear and soft sectors factorize.

${ }^{6}$ In fact, since at higher loop order the number of factors of $\gamma_{\perp}^{\nu_{i}}$ may not decrease, the matrix of renormalization constants is block triangular, with $Z_{n m}=0$ for $n<m$. We do not make use of this fact in the present discussion.
} 
point, the elements of the anomalous dimension matrix describing the mixing of the physical operator into evanescents vanish. Although we have specialized here to the case of the scalar current, the arguments may be easily extended to the general case.

The basis of evanescent operators $O_{n}$ is of course not unique. We could for instance have chosen the antisymmetrized basis,

$$
\left[\hat{O}_{n}\right]_{a b}=\left[\bar{X} \gamma_{\perp}^{\left[\nu_{1}\right.} \cdots \gamma_{\perp}^{\left.\nu_{2 n+1}\right]}\right]_{a}\left[\gamma_{\perp\left[\nu_{1}\right.} \cdots \gamma_{\left.\perp \nu_{2 n+1}\right]} X\right]_{b}
$$

or any other basis which is related to the original basis of operators by

$$
O_{0}=\hat{O}_{0} \quad \text { and } \quad O_{n}=\sum_{m=1}^{n} A_{n m} \hat{O}_{m}+\epsilon B_{n} \hat{O}_{0} \text { for } n \geq 1,
$$

with coefficients $A_{n m}, B_{n}$, finite at $d=4$. The same renormalized operators may be expressed in terms of the new basis of bare operators as

$$
\left(\begin{array}{c}
O_{0}^{\text {ren }} \\
O_{1}^{\text {ren }} \\
O_{2}^{\text {ren }} \\
\vdots
\end{array}\right)=\left(\begin{array}{cccc}
Z_{00}+\epsilon \sum_{m=1}^{\infty} Z_{0 m} B_{m} & \sum_{m=1}^{\infty} Z_{0 m} A_{m 1} & \sum_{m=2}^{\infty} Z_{0 m} A_{m 2} \cdots \\
\epsilon \sum_{m=1}^{\infty} Z_{1 m} B_{m} & \sum_{m=1}^{\infty} Z_{1 m} A_{m 1} & \sum_{m=2}^{\infty} Z_{1 m} A_{m 2} & \cdots \\
\epsilon \sum_{m=1}^{\infty} Z_{2 m} B_{m} & \sum_{m=1}^{\infty} Z_{2 m} A_{m 1} & \sum_{m=2}^{\infty} Z_{2 m} A_{m 2} & \cdots \\
\vdots & \vdots & \vdots & \ddots
\end{array}\right)\left(\begin{array}{c}
\hat{O}_{0}^{\text {bare }} \\
\hat{O}_{1}^{\text {bare }} \\
\hat{O}_{2}^{\text {bare }} \\
\vdots
\end{array}\right) .
$$

If we identify the resulting matrix with the renormalization constant matrix $\hat{\mathbf{Z}}$ for the new basis, then we find for $n \geq 1$ the typical counterterms, $\hat{Z}_{n 0}=\epsilon \sum_{m=1}^{\infty} Z_{n m} B_{m}$, involving finite constants which must be included in order to ensure the vanishing of the renormalized evanescent operators. We also find, however, a finite contribution to $\hat{Z}_{00}=Z_{00}+\epsilon \sum_{m=1}^{\infty} Z_{0 m} B_{m}$. If, as is customary, we impose the $\overline{\mathrm{MS}}$ scheme for the physical operators and allow finite subtractions only for evanescent operators, in the terms $\hat{Z}_{n 0}$ for $n \geq 1$, then the finite contribution to $\hat{Z}_{00}$ must be absorbed into the Wilson coefficient function of $O_{0}^{\text {ren }}$. Since this finite contribution depends on the choice of basis in (42), a scheme dependence is induced in the coefficient functions of the operators; this scheme dependence cancels against the corresponding scheme dependence in the operator matrix elements.

\subsection{Scheme dependence of the jet functions}

From the preceding discussion, we find that using the $\overline{\mathrm{MS}}$ scheme for the physical operators, a change of evanescent operator basis corresponds to a change of renormalization scheme. In this section we work in the opposite direction, and ask if it is possible to find a basis of evanescent operators which yields a given renormalization scheme for the physical operators. In particular, when we take the matrix elements of physical operators describing form factors, we would like the LCDAs appearing in (1) to be defined in the $\overline{\mathrm{MS}}$ scheme.

We start by defining a Fierz rearranged operator $O_{i}^{\prime}$ for each physical operator $O_{i}$ in such a way that the bare operators coincide in four dimensions:

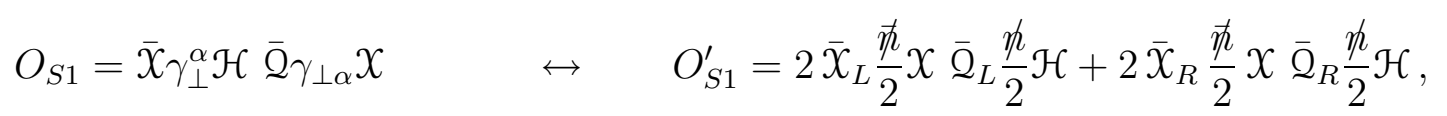




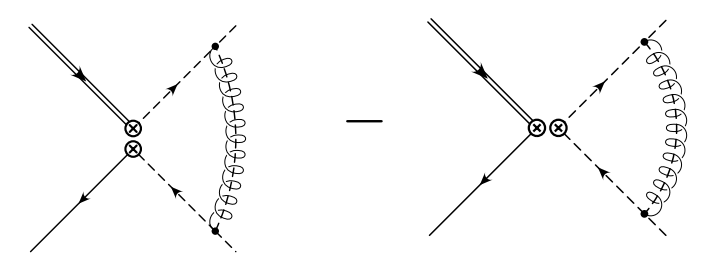

Figure 5: One-loop graphs contributing to the difference between the matrix element of the original and the Fierz rearranged four-quark operators.

$$
\begin{aligned}
& O_{V 3}=\bar{X}_{\gamma_{\perp}^{\mu}}^{\mu} \gamma_{\perp}^{\alpha} \mathcal{H} \overline{\mathcal{Q}} \gamma_{\perp \alpha} X \quad \leftrightarrow \quad O_{V 3}^{\prime}=2 \bar{X}_{L} \gamma_{\perp}^{\mu} \frac{\not h}{2} X \overline{\mathcal{Q}}_{R} \frac{\not h}{2} \mathcal{H}+(L \leftrightarrow R) \\
& O_{V 4}=\bar{X}_{\gamma_{\perp}^{\alpha}}^{\alpha} \gamma_{\perp}^{\mu} \mathcal{H} \overline{\mathcal{Q}} \gamma_{\perp \alpha} X \quad \leftrightarrow \quad O_{V 4}^{\prime}=2 \bar{X}_{L} \frac{\not h}{2} X \overline{\mathcal{Q}}_{L} \frac{\not h}{2} \gamma_{\perp}^{\mu} \mathcal{H}+(L \leftrightarrow R), \\
& O_{T 6}=\bar{X}_{\gamma_{\perp}^{\alpha}}^{\alpha} \gamma_{\perp}^{\mu \nu} \mathcal{H} \overline{\mathcal{Q}}_{\gamma_{\perp \alpha}} \chi \quad \leftrightarrow \quad O_{T 6}^{\prime}=2 \bar{X}_{L} \frac{\hbar}{2} \chi \overline{\mathcal{Q}}_{L} \frac{\not h}{2} \gamma_{\perp}^{\mu \nu} \mathcal{H}+(L \leftrightarrow R),
\end{aligned}
$$

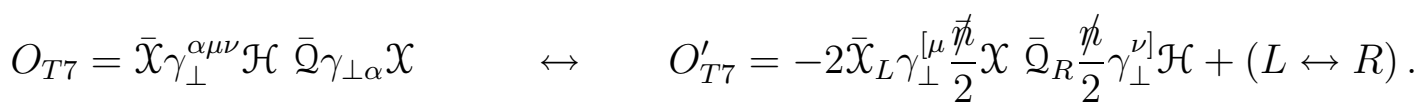

The remaining physical vector and tensor operators are obtained by multiplying the above operators by factors of $v^{\mu}$ and $n^{\mu}$. In fact, since $O_{V 4}$ and $O_{T 6}$ are obtained from $O_{S 1}$ by insertion of Dirac structures next to the heavy quark field, these operators don't require an independent analysis. Similarly, from the identity $-2 \gamma_{\perp}^{\alpha \mu \nu}=\gamma_{\perp}^{\mu} \gamma_{\perp}^{\alpha} \gamma_{\perp}^{\nu}-\gamma_{\perp}^{\nu} \gamma_{\perp}^{\alpha} \gamma_{\perp}^{\mu}$, we may relate $O_{T 7}$ to $O_{V 3}$. It is convenient to treat $O_{T 7}$ in the same manner as the other operators in (44), even though it is evanescent. We will find that the resulting renormalization scheme automatically ensures the vanishing of the renormalized $O_{T 7}$.

We define the renormalized physical operators $O_{i}$ and $O_{i}^{\prime}$ in the $\overline{\mathrm{MS}}$ scheme. The heavy-tolight meson matrix elements of the Fierz rearranged operators $O_{i}^{\prime}$ will then yield the product of the LCDAs of the two mesons in the $\overline{\mathrm{MS}}$ scheme. Our task is to define the evanescent operators in such a way that physical matrix elements are the same for $O_{i}$ and $O_{i}^{\prime}$. For instance, if we consider a four-point Green function involving $O_{S 1}^{\prime}$, then at one-loop there are contributions proportional to the tree-level structure, as well as a contribution proportional to

$$
\frac{1}{\epsilon} \times \gamma_{\perp}^{\alpha} \gamma_{\perp}^{\beta} \frac{\not h}{2} \gamma_{\perp \beta} \gamma_{\perp \alpha}=\frac{1}{\epsilon} \times\left(4-8 \epsilon+4 \epsilon^{2}\right) \frac{\not h}{2} \rightarrow-8 \frac{\not \hbar}{2} .
$$

This contribution arises from the second diagram in Figure 5. In the $\overline{\mathrm{MS}}$ scheme, only the $1 / \epsilon$ pole is subtracted to obtain the renormalized result. Similarly, the matrix element of $O_{S 1}$ depicted in the first diagram in Figure 5 contains the same contribution proportional to the tree-level structure $\gamma_{\perp}^{\alpha} \otimes \gamma_{\perp \alpha}$, and a contribution proportional to

$$
\begin{aligned}
\frac{1}{\epsilon} \times \gamma_{\perp}^{\alpha} \gamma_{\perp}^{\beta} \gamma_{\perp}^{\gamma} \otimes \gamma_{\perp \gamma} \gamma_{\perp \beta} \gamma_{\perp \alpha} & =\frac{1}{\epsilon} \times(4-6 \epsilon) \gamma_{\perp}^{\alpha} \otimes \gamma_{\perp \alpha}-\frac{1}{\epsilon} \times \gamma_{\perp}^{\alpha \beta \gamma} \otimes \gamma_{\perp \alpha \beta \gamma} \\
& \rightarrow-6 \gamma_{\perp}^{\alpha} \otimes \gamma_{\perp \alpha} .
\end{aligned}
$$


Using $\overline{\mathrm{MS}}$ subtraction in the original basis (26) yields a renormalized result different from the corresponding result in (45). In place of (46), we should instead write

$$
\begin{aligned}
& \frac{1}{\epsilon} \times \gamma_{\perp}^{\alpha} \gamma_{\perp}^{\beta} \gamma_{\perp}^{\gamma} \otimes \gamma_{\perp \gamma} \gamma_{\perp \beta} \gamma_{\perp \alpha} \\
& =\frac{1}{\epsilon} \times\left(4-8 \epsilon+4 \epsilon^{2}\right) \gamma_{\perp}^{\alpha} \otimes \gamma_{\perp \alpha}-\frac{1}{\epsilon} \times\left[\gamma_{\perp}^{\alpha \beta \gamma} \otimes \gamma_{\perp \alpha \beta \gamma}-2 \epsilon(1-2 \epsilon) \gamma_{\perp}^{\alpha} \otimes \gamma_{\perp \alpha}\right] \\
& \rightarrow-8 \gamma_{\perp}^{\alpha} \otimes \gamma_{\perp \alpha} .
\end{aligned}
$$

The resulting contributions to the renormalized matrix elements of $O_{S 1}^{\prime}$ and $O_{S 1}$ will therefore agree if we choose in place of $O_{S 2}$ in (26),

$$
\hat{O}_{S 2}=O_{S 2}-2 \epsilon(1-2 \epsilon) O_{S 1} \text {. }
$$

Beyond one-loop, the other evanescent operators $\hat{O}_{S 3}, \hat{O}_{S 4}, \ldots$ are likewise determined. Proceeding in the same way for the vector case, we find

$$
\begin{aligned}
& \hat{O}_{V 7}=O_{V 7}+2 \epsilon(1+2 \epsilon) O_{V 3}-4 \epsilon O_{V 4}, \\
& \hat{O}_{V 8}=O_{V 8}-2 \epsilon(1-2 \epsilon) O_{V 4} .
\end{aligned}
$$

Dropping the $\mathcal{O}\left(\epsilon^{2}\right)$ terms which are irrelevant through one-loop order, the scalar and vector jet-functions in this new basis are (cf. (29))

$$
\begin{aligned}
& \hat{\mathcal{J}}_{S}=\begin{array}{c}
J_{S} \\
\hat{O}_{S 2}
\end{array}\left(\begin{array}{c}
\mathcal{J}_{1}+2(1-\epsilon) \mathcal{J}_{2}+2 \epsilon \mathcal{J}_{3} \\
\mathcal{J}_{3}
\end{array}\right) \\
& \hat{\mathcal{J}}_{V}^{\perp}=\begin{array}{cc}
J_{V 1}^{B^{\prime}} & J_{V 4}^{B^{\prime}} \\
O_{V 3} \\
O_{V 4} \\
\hat{O}_{V 7} \\
\hat{O}_{V 8}
\end{array}\left(\begin{array}{cc}
\mathcal{J}_{1}+2(1-\epsilon) \mathcal{J}_{2}+2 \epsilon \mathcal{J}_{3} & 2 \epsilon \mathcal{J}_{2}+4 \epsilon \mathcal{J}_{3} \\
0 & \mathcal{J}_{3} \\
\mathcal{J}_{3} & 0
\end{array}\right) .
\end{aligned}
$$

Although they will not be relevant to the form factor analysis, for completeness we give here also the new basis of evanescent operators for the tensor case,

$$
\begin{aligned}
& \hat{O}_{T 13}=O_{T 13}-2 \epsilon(1-2 \epsilon) O_{T 6}, \\
& \hat{O}_{T 14}=O_{T 14}+2 \epsilon(1+2 \epsilon) O_{T 7}+4 \epsilon O_{T 6},
\end{aligned}
$$

and the corresponding jet functions,

$$
\hat{\mathcal{J}}_{T}^{\perp \perp}=\begin{gathered}
J_{T 1}^{B^{\prime}} \\
O_{T 6} \\
O_{T 7} \\
\hat{O}_{T 13} \\
\hat{O}_{T 14}
\end{gathered}\left(\begin{array}{cc}
\mathcal{J}_{1}+2(1-\epsilon) \mathcal{J}_{2}+2 \epsilon \mathcal{J}_{3} & -2 \epsilon \mathcal{J}_{2}-4 \epsilon \mathcal{J}_{3} \\
0 & \mathcal{J}_{1}-2 \epsilon \mathcal{J}_{3} \\
\mathcal{J}_{3} & 0 \\
0 & \mathcal{J}_{3}
\end{array}\right) .
$$


We may recall from (44) that the operators $O_{T 7}$ and $O_{T 7}^{\prime}$ vanish at tree level in $d=4$. In fact, since it is multiplicatively renormalized, the renormalized $O_{T 7}^{\prime}$ then vanishes to all orders. Using the new basis of evanescents, $\hat{O}_{T 13}, \hat{O}_{T 14}, \ldots$, the same is true for $O_{T 7}$, since the new basis was chosen precisely to ensure that renormalized matrix elements of $O_{T 7}$ and $O_{T 7}^{\prime}$ agree. In this scheme, therefore, $O_{T 6}$ is identified with the renormalized physical operator, and the renormalized $O_{T 7}$ vanishes. It is interesting to note that the evanescent $J_{T 5}^{B^{\prime}}$ gives a finite contribution at one-loop order to the bare matching coefficient of the physical operator $O_{T 6}$ in (53). As discussed in Section 4.1, since we use the $\overline{\mathrm{MS}}$ scheme for all operators in $\mathrm{SCET}_{\mathrm{I}}$, the corresponding matching coefficient between renormalized operators will also be nonzero.

Let us illustrate the above arguments by an explicit evaluation of the diagrams in Figure 5 for the scalar case. In terms of tree-level matrix elements, the difference between the renormalized matrix elements at one-loop order is

$$
\begin{aligned}
\left\langle O_{S 1}^{\mathrm{ren}}(u)\right\rangle\left(u^{\prime}\right)-\left\langle O_{S 1}^{\prime \mathrm{ren}}(u)\right\rangle\left(u^{\prime}\right) & =\int d v \Delta\left(v, u^{\prime}\right)\left[(4-6 \epsilon)\left\langle O_{S 1}(u)\right\rangle(v)-\left\langle O_{S 2}(u)\right\rangle(v)\right. \\
& \left.-4(1-\epsilon)^{2}\left\langle O_{S 1}^{\prime}(u)\right\rangle(v)\right]+\int d v Z_{S 1, S 2}(u, v)\left\langle O_{S 2}(v)\right\rangle\left(u^{\prime}\right) .
\end{aligned}
$$

Here $u$ is the Fourier transform variable in (18) and $u^{\prime}$ is the momentum fraction of the outgoing collinear quark in the diagrams in Figure 5. At tree level, we have $\left\langle O_{i}(u)\right\rangle\left(u^{\prime}\right) \propto \delta\left(u-u^{\prime}\right)$. The function $\Delta$ is given by

$$
\Delta\left(u, u^{\prime}\right)=\frac{\alpha_{s} C_{F}}{4 \pi} \frac{1}{2 \epsilon}\left[\frac{u}{u^{\prime}} \theta\left(u^{\prime}-u\right)+\frac{\bar{u}}{\bar{u}^{\prime}} \theta\left(u-u^{\prime}\right)\right]=\frac{\alpha_{s}}{4 \pi} \frac{(-\bar{u})}{\bar{u}^{\prime}} J_{3}^{(1)}\left(u^{\prime}, u\right)+O\left(\epsilon^{0}\right),
$$

where in the last equality we compare to the explicit form of $J_{3}^{(1)}$ in (24). Since the divergences have to cancel, we see that $\Delta=Z_{S 1, S 2}$, and in our original evanescent operator basis the finite difference in the renormalized matrix elements becomes

$$
\left\langle O_{S 1}^{\mathrm{ren}}(u)\right\rangle\left(u^{\prime}\right)-\left\langle O_{S 1}^{\prime \mathrm{ren}}(u)\right\rangle\left(u^{\prime}\right)=\int d v 2 \epsilon \Delta\left(v, u^{\prime}\right)\left\langle O_{S 1}(u)\right\rangle(v) .
$$

The one-loop Wilson coefficient functions of these operators must therefore fulfill the relation

$$
\mathcal{J}_{S 1}^{\prime}(u, v)-\mathcal{J}_{S 1}(u, v)=\int d y \mathcal{J}_{S 1}^{\text {tree }}(y, v) 2 \epsilon \Delta(y, u)=2 \epsilon \mathcal{J}_{3}(u, v)
$$

which is in agreement with $(29)$ and $(50)$, since $\hat{\mathcal{J}}_{S 1}=\mathcal{J}_{S 1}^{\prime}$.

Having determined the operator basis, we now renormalize the jet-functions. After $\overline{\mathrm{MS}}$ subtractions, the quantities $\mathcal{J}_{1}+2(1-\epsilon) \mathcal{J}_{2}+2 \epsilon \mathcal{J}_{3}$ and $\mathcal{J}_{1}-2 \epsilon \mathcal{J}_{3}$ yield $\mathcal{J}_{\|}$and $\mathcal{J}_{\perp}$ from [16] respectively. We recall that $\mathcal{J}_{\|}$is the jet function appearing in all form factors describing $B$ decays into pseudoscalar mesons, or longitudinally-polarized vector mesons, while $\mathcal{J}_{\perp}$ appears for all perpendicularly-polarized vector mesons. In [16] we introduced the following notation,

$$
\mathcal{J}_{\|, \perp}(u, v, L, \mu)=\frac{4 \pi C_{F} \alpha_{s}(\mu)}{N} \frac{1}{2 E \bar{u}}\left[-\delta(u-v)+\frac{\alpha_{s}(\mu)}{4 \pi} j_{\|, \perp}(u, v, L)+\mathcal{O}\left(\alpha_{s}^{2}\right)\right],
$$


where $L=\ln \left(2 E \omega / \mu^{2}\right), j_{\|}=j_{1}+j_{2}+j_{3}$ and $j_{\perp}=j_{1}-j_{3}$. In terms of the one-loop expressions (22) and (23), after $\overline{\mathrm{MS}}$ subtractions we have

$$
\begin{aligned}
J_{1}^{(1)} & \rightarrow j_{1} \\
2(1-\epsilon) J_{2}^{(1)} & \rightarrow j_{2} \\
2 \epsilon J_{3}^{(1)} & \rightarrow j_{3} .
\end{aligned}
$$

The quantity $2 \epsilon \mathcal{J}_{2}$ also appears, and we find

$$
\begin{aligned}
2 \epsilon J_{2}^{(1)}(u, v) \rightarrow 2 & \left(C_{F}-\frac{C_{A}}{2}\right)\left[\frac{\bar{u}}{u \bar{v}} \theta(u-v)+\frac{\bar{v}}{u \bar{u}} \theta(v-u)\right. \\
& \left.+\frac{u v-(1-u-v)^{2}}{u \bar{u} \bar{v}} \theta(1-u-v)\right]+2 C_{F}\left[\frac{v-u}{u \bar{u}} \theta(v-u)-\frac{v \bar{u}}{u}\right] .
\end{aligned}
$$

However, this jet function occurs only in the matching onto operators irrelevant to the form factor analysis. Similarly, the quantity $\mathcal{J}_{3}$ appears in the matching onto evanescents.

\section{Application to heavy-to-light form factors}

In this section we study the numerical size of the matching corrections in the factorization formula for the form factors. To begin, we review the situation for the $A$-type currents. This case is especially simple because no convolution over momentum fractions is involved, so that no assumptions on the shape of hadronic wave functions are necessary in order to make statements about the size of one-loop corrections relative to the tree-level values. We consider the combinations of Wilson coefficients for the $A$-type currents which yield the $C_{i}$ in the first term of (1) for the form factors $F_{+}, F_{0}, F_{T}, V$ and $T_{1}$. To distinguish these from the $C_{i}^{B}$ in the hard-scattering terms, we will denote them by $C_{i}^{A}$ in the following. Form factor normalizations are chosen such that $C_{i}^{A}$ is equal to unity at tree level. The coefficients for the form factors of longitudinally-polarized vector mesons $\left(A_{0}, V-A_{2}, T_{2}-\left(2 E / m_{B}\right) T_{3}\right)$ are identical to the pseudoscalar case. Also, for perpendicularly-polarized vector mesons, form factors $V$ and $T_{2}$ are related to $A_{1}$ and $T_{1}$ respectively. The same relations also hold for the hard-scattering terms. Using the expressions from [5, 17], we find that the one-loop corrections are remarkably similar for all form factors. As illustrated in the plot in Figure 5, the corrections are negative, ranging in size from approximately $10-15 \%$ at $E=m_{b} / 2$ to $20-25 \%$ at $E=m_{b} / 4$. The symmetry breaking part of these corrections is very small, less than $5 \%$ in the energy range considered. This may indicate that the bulk of the one-loop correction is simply due to the choice of renormalize scheme or factorization scale.

The analysis is more complicated for the $B$-type current operators. Since the hardscattering kernel $T_{i}(E, \omega, u)$ appearing in the factorization formula (1) depends on variables $\omega$ and $u$, the numerical impact of the loop corrections can be assessed only after convolution over these variables. Knowledge of the wavefunctions of the $B$-meson and light final state 


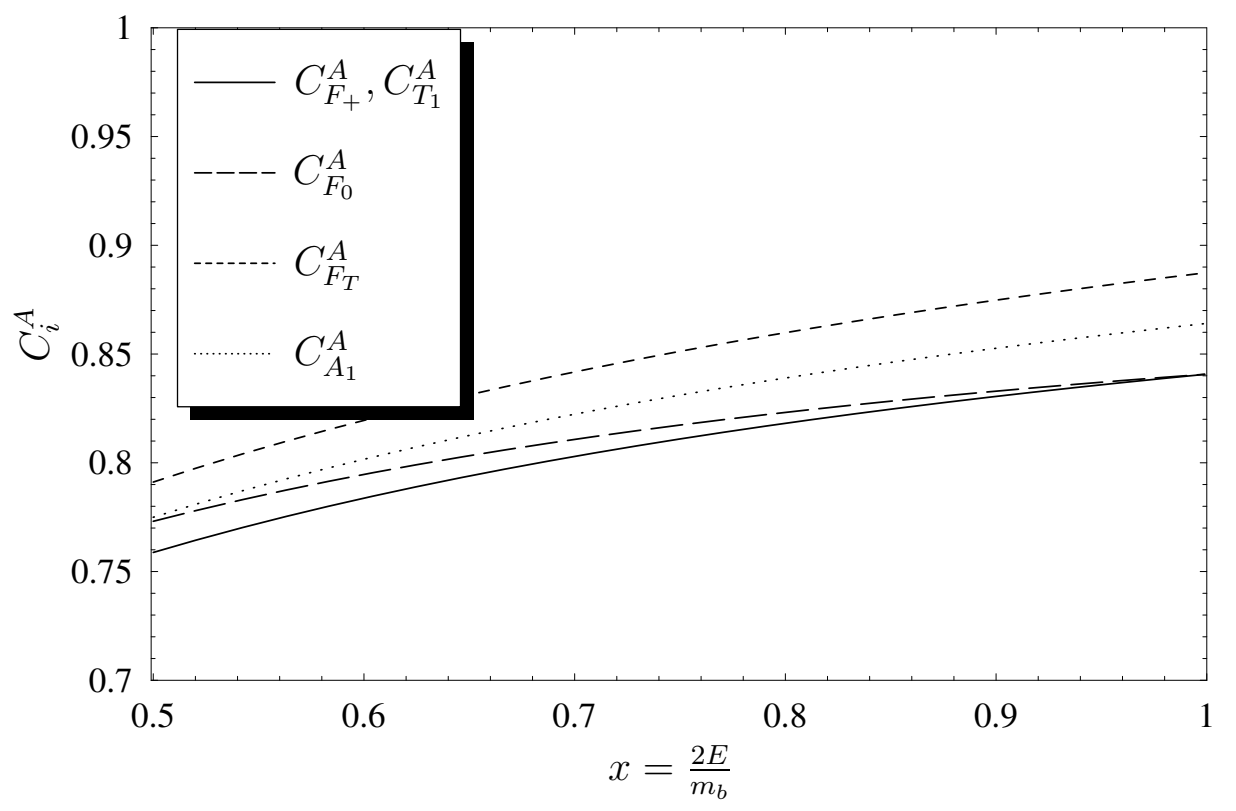

Figure 6: One-loop coefficients $C_{i}^{A}(E)$ of the soft-overlap terms in the heavy-to-light form factors as a function of the recoil energy. The QCD as well as the effective theory renormalization scale are set equal to $m_{b}$, and we take $\alpha_{s}\left(m_{b}\right)=0.22$. All coefficients are equal to one at tree level.

meson appearing in the convolution is therefore necessary. To begin, we recall the form of the hard-scattering term [16]

$$
\begin{aligned}
\Delta F_{i}=\frac{m_{B}}{2 E} \frac{f_{B}}{4 K_{F}(\mu)} \int_{0}^{\infty} \frac{d \omega}{\omega} \phi_{B}(\omega, \mu) \int_{0}^{1} d u f_{M}(\mu) \phi_{M}(u, \mu) \\
\qquad \int_{0}^{1} d v \mathcal{J}_{\Gamma}\left(u, v, \ln \frac{2 E \omega}{\mu^{2}}, \mu\right) C_{i}^{B}(E, v, \mu),
\end{aligned}
$$

where $\mathcal{J}_{\Gamma}=\mathcal{J}_{\|}$for pseudoscalar and longitudinally-polarized vector mesons and $\mathcal{J}_{\Gamma}=\mathcal{J}_{\perp}$ for perpendicularly polarized vector mesons. The quantity $K_{F}(\mu)=1+\frac{C_{F} \alpha_{s}}{4 \pi}\left(3 \ln \frac{m_{b}}{\mu}-2\right)$ relates the QCD and the HQET $B$-meson decay constants. In order to avoid large perturbative logarithms in the coefficients $C_{i}^{B}$, one would like to set $\mu^{2} \sim m_{b}^{2}$, whereas the scale associated with the jet-function is lower, $\mu \sim \sqrt{m_{b} \Lambda_{h}}$, where $\Lambda_{h}$ is a typical hadronic scale. Since the form factor involves different scales, we see that we cannot avoid large perturbative logarithms in a fixed order calculation. These logarithms can be resummed by solving the renormalization group equations for the Wilson coefficients. This is discussed in detail in [16], where we resummed all leading logarithms of the different energy scales in the problem. The two effects we wish to study here are the one-loop corrections to $C_{i}^{B}$, and to $\mathcal{J}_{\Gamma}$. We study the two corrections in turn, evaluating each of them at their natural scale $\mu$, but remind the reader that such a scale choice is strictly only possible after renormalization group improvement.

Let us start by considering the hard-scale matching corrections to the $B$-type Wilson 
coefficients. The five independent combinations appearing in the form factors are

$$
\begin{aligned}
C_{F_{+}}^{B} & =\frac{E}{m_{b}} C_{V 2}^{B^{\prime}}+C_{V 3}^{B^{\prime}}=1-\frac{4 E}{m_{b}}+\ldots, \\
C_{F_{0}}^{B} & =\left(1-\frac{E}{m_{b}}\right) C_{V 2}^{B^{\prime}}+C_{V 3}^{B^{\prime}}=-1+\ldots, \\
C_{F_{T}}^{B} & =\frac{1}{2} C_{T 4}^{B^{\prime}}=1+\ldots, \\
C_{A_{1}}^{B} & =C_{V 4}^{B^{\prime}}=0+\ldots, \\
C_{T_{1}}^{B} & =-\frac{1}{2}\left(1-\frac{E}{m_{b}}\right) C_{T 6}^{B^{\prime}}-\frac{1}{2} C_{T 7}^{B^{\prime}}=-\frac{2 E}{m_{b}}+\ldots
\end{aligned}
$$

At tree level they take the values indicated; the pertinent one-loop expressions were given in Section 2. To evaluate the impact of these corrections on the form factors we work with the tree-level jet-function, which is independent of $\omega$ :

$$
\mathcal{J}_{\Gamma}(u, v)=-\frac{4 \pi C_{F} \alpha_{s}(\mu)}{N} \frac{1}{2 E \bar{u}} \delta(u-v)+\mathcal{O}\left(\alpha_{s}^{2}\right)
$$

The size of the hard-matching corrections is then determined by convoluting the Wilson coefficient $C_{i}^{B}$ with the light-meson wave function. Using for simplicity the asymptotic form $\phi_{M}(u)=6 u \bar{u}$ of the light meson LCDA, the relevant integral is

$$
C_{i}^{B(\mathrm{eff})}(E, \mu)=\frac{2}{K_{F}(\mu)} \int_{0}^{1} d u u C_{i}^{B}(E, u, \mu) .
$$

These effective coefficients coincide with the $C_{i}^{B}$ at tree level, since dependence on the momentum fraction $u$ appears only at one-loop order. In terms of $C_{i}^{B(\text { eff })}$ the correction to the form-factor is

$$
\Delta F_{i}=-\left(\frac{m_{B}}{2 E}\right)^{2}\left[\frac{3 \pi C_{F} \alpha_{s}(\mu)}{N} \frac{f_{B} f_{M}(\mu)}{m_{B} \lambda_{B}(\mu)}\right] C_{i}^{B(\mathrm{eff})}(E, \mu),
$$

where $\lambda_{B}(\mu)$ is defined as the first inverse moment of the $B$-meson LCDA,

$$
\lambda_{B}^{-1}(\mu) \equiv \int_{0}^{\infty} \frac{d \omega}{\omega} \phi_{B}(\omega, \mu)
$$

The quantity inside square brackets in (65) coincides, up to RG factors, with the tree-level value of the function $H_{M}$ introduced in [16]. In Figure 7, we plot the one-loop contributions to $C_{i}^{B(\text { eff })}(E, \mu)$. Choosing the scale $\mu=m_{b}$, we find that the one-loop corrections are of order $20 \%$ of the tree-level values given in (62).

Finally, to isolate the one-loop corrections from the jet functions, we use tree-level matching for the hard coefficients. Since these tree-level coefficients are independent of the momentum fraction $u$, in this approximation the resulting convolution integrals in (61) are universal to 


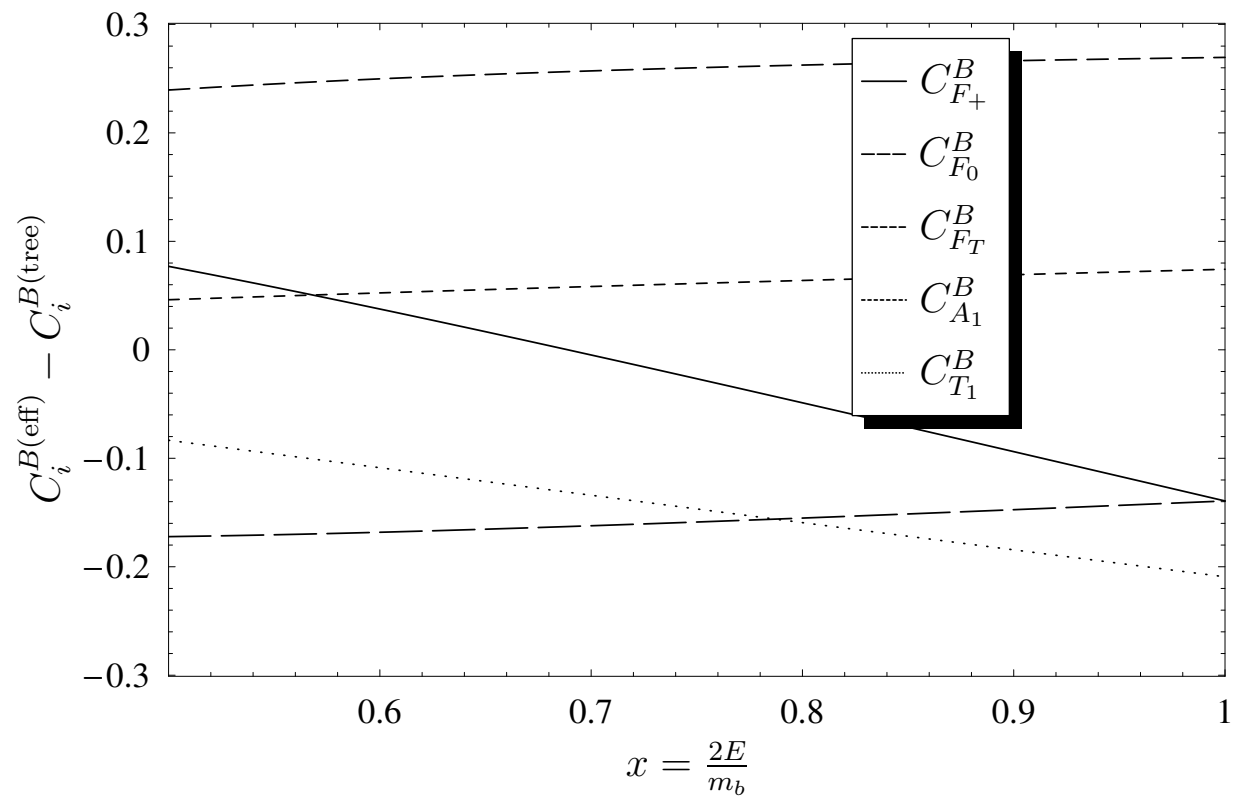

Figure 7: One-loop corrections to the form factors from the Wilson coefficients $C_{i}^{B}(E, u)$. The figure shows the one-loop correction to the quantity $C_{i}^{B(\mathrm{eff})}(E)$ that arises after convolution of the coefficient $C_{i}^{B}(E, u)$ with the leading order jet-function and the asymptotic pion LCDA, see (64). The renormalization scale is set equal to $m_{b}$ in both QCD and the effective theory.

all form factors involving the same final-state meson. For pseudoscalar and longitudinallypolarized vector mesons, the result is proportional to [16]

$$
\begin{aligned}
& \int_{0}^{\infty} \frac{d \omega}{\omega} \phi_{B}(\omega, \mu) \int_{0}^{1} d u \phi_{M}(u, \mu) \int_{0}^{1} d v \mathcal{J}_{\|}\left(u, v, \ln \left(2 E \omega / \mu^{2}\right), \mu\right) \\
& \propto 1+\frac{\alpha_{s}(\mu)}{4 \pi}\left[\frac{4}{3}\left\langle\ln ^{2} \frac{2 E \omega}{\mu^{2}}\right\rangle+\left(-\frac{19}{3}+\frac{\pi^{2}}{9}\right)\left\langle\ln \frac{2 E \omega}{\mu^{2}}\right\rangle+3.99\right],
\end{aligned}
$$

where for simplicity we have again used the asymptotic form for the light-meson LCDAs. We take $n_{f}=4$ as the number of light quark flavors. For perpendicularly-polarized vector mesons, where $\mathcal{J}_{\|}$is replaced by $\mathcal{J}_{\perp}$, the coefficient of the double logarithm is the same as in (67), the coefficient of the single logarithm is changed from $\left(-19 / 3+\pi^{2} / 9\right)$ to $\left(-6+\pi^{2} / 9\right)$, and the constant term is changed from 3.99 to 0.73 . Here the angle brackets denote averages over the $B$-meson LCDA,

$$
\langle g(\omega)\rangle \equiv \lambda_{B}(\mu) \int_{0}^{\infty} \frac{d \omega}{\omega} \phi_{B}(\omega, \mu) g(\omega) .
$$

To investigate the size of the one-loop corrections, we may take illustrative models for the $B$-meson LCDA. For example, in [25],

$$
\phi_{B}\left(\omega, \mu_{0}\right)=\frac{\omega}{\lambda_{B}^{2}} e^{-\omega / \lambda_{B}}, \quad \lambda_{B}=\frac{2}{3}\left(m_{B}-m_{b}\right) \approx 0.32 \mathrm{GeV},
$$


where $\mu_{0}$ is a low hadronic scale at which the model is assumed valid. For the averages in (67), we evaluate the wavefunction appearing in (68) at the scale $\mu_{0}$, and take the intermediate scale $\mu_{i}^{2}=m_{b} \Lambda_{h}$, with $\Lambda_{h}=0.5 \mathrm{GeV}$, as the argument of the jet function. For maximal energy $E=m_{b} / 2$ this model yields

$$
\begin{aligned}
\left\langle\ln \frac{2 E \omega}{\mu_{i}^{2}}\right\rangle & =\ln \left(\frac{2 E \lambda_{B} \exp ^{-\gamma_{E}}}{\mu_{i}^{2}}\right) \sim-1.0 \\
\left\langle\ln ^{2}\left(\frac{2 E \omega}{\mu_{i}^{2}}\right)\right\rangle & =\ln ^{2}\left(\frac{2 E \lambda_{B} \exp ^{-\gamma_{E}}}{\mu_{i}^{2}}\right)+\frac{\pi^{2}}{6} \sim 2.7 .
\end{aligned}
$$

The resulting hard-scattering corrections in (67) are approximately $+30 \%$ and $+40 \%$ for $\mathcal{J}_{\|}$and $\mathcal{J}_{\perp}$ respectively. Neglecting the implicit model-dependence of these results, the uncertainty due to $m_{b}$ and hence of $\lambda_{B}$ is still significant; for instance, taking $\lambda_{B}=0.46 \mathrm{GeV}$ [26] reduces the size of corrections to approximately $+20 \%$ and $+30 \%$. Using the model wavefunction from a more recent analysis in [26], with the same value of the intermediate scale in the jet function, and at maximal energy $E=m_{b} / 2$, gives

$$
\left\langle\ln \frac{2 E \omega}{\mu_{i}^{2}}\right\rangle \sim-0.7 \pm 0.4, \quad\left\langle\ln ^{2}\left(\frac{2 E \omega}{\mu_{i}^{2}}\right)\right\rangle \sim 1.0 \pm 0.6
$$

where the wavefunction is evaluated at the scale $1 \mathrm{GeV}$. In this model, the corrections for $\mathcal{J}_{\|}$ and $\mathcal{J}_{\perp}$ are approximately $15 \%$ and $25 \%$ at $E=m_{b} / 2$, and grow larger at small values of $E$, reaching $30 \%$ and $40 \%$ at $E=m_{b} / 4$. In evaluating the averages in (67) with wavefunctions evaluated at the low scale $\mu \sim 1 \mathrm{GeV}$, and at the same time taking $\mu_{i}=m_{b} \Lambda_{h}$ as the argument of the jet functions, we have neglected the RG factors relating the scales $\mu$ and $\mu_{i}$. From [16], we find the resulting correction to (67) for this choice of scales to be approximately $-15 \%$.

\section{Discussion and conclusions}

We have presented in this paper the complete results for the one-loop matching corrections to the hard-scattering coefficients in the factorization formula (1) which describes heavy-to-light form factors at large recoil. Our results (9) and (10) for the hard-scale matching coefficients $C_{i}^{B}$ in the vector and tensor case extend our previous scalar current results reported in [16], and are in agreement with an independent calculation [17]. Results for the intermediate-scale coefficients, $\mathcal{J}_{\|}$and $\mathcal{J}_{\perp}$ in (58), which are relevant to $B$ decay form factors were reported in [16]; in this paper we have presented general results, and details of the calculation.

An interesting theoretical issue involves the evanescent operators which arise upon matching QCD onto the effective theory. We showed that even in the presence of divergences of the Sudakov type, a renormalization scheme exists for which renormalized evanescent operators vanish. The issue arises already in $\mathrm{SCET}_{\mathrm{I}}$, for the $B$-type operators, and we discussed this case first. This example is especially interesting since the mixing involves only two operators, in contrast to the usual infinite tower of evanescent operators induced by radiative corrections to four-fermion interactions. The case of the nonlocal four-quark operators of $\mathrm{SCET}_{\mathrm{II}}$ was then treated along the same lines. On a practical level, use of such a renormalization 
scheme implies that matching coefficients onto evanescent operators become irrelevant below the matching scale, since the renormalized operator matrix elements vanish, and the physical operators do not mix into the evanescents. We also discussed the choice of evanescent operator basis. Different bases correspond to different renormalization schemes for the physical operators. We isolated a particular basis which corresponds to the $\overline{\mathrm{MS}}$ scheme after Fierz transformation.

We have presented an analysis of the phenomenological impact of one-loop radiative corrections in Section 5. Using the known results for the $A$-type currents [5, 17], we find that the matching corrections to the soft-overlap terms are remarkably universal to all form factors. The corrections are of order $15 \%$, but differ in all cases by less than $5 \%$. This effect seems not to have been noticed previously in the literature, and implies that any breaking of the naive large-energy spin-symmetry relations by more than $5 \%$ is due to either the hard-scattering contributions, or to uncalculated power corrections. We have also investigated the numerical impact of radiative corrections to the hard-scattering coefficients, finding the hard-scale corrections to be $\lesssim 20 \%$ for all form factors. These corrections determine the size of violations to the universality of the hard-scattering terms for different form factors which holds at tree level for the hard-scale coefficient. Radiative corrections to the jet functions are less important from the point of view of universality, since they contribute identically to all form factors involving the same final state meson. On the other hand, for the purpose of calculating the hard-scattering contributions in terms of the $B$ meson wavefunction, the jet function corrections are expected to be more important than the hard-scale corrections, since the coupling constant $\alpha_{s}(\mu)$ is larger at the intermediate scale $\mu^{2} \sim \Lambda_{\mathrm{QCD}} m_{b}$. For this reason it has been speculated that the jet-functions might not have a convergent perturbative expansion for physical values of $m_{b}$ [27]. We evaluated the perturbative corrections to the jet-functions for two models of $\phi_{B}(\omega)$, finding that one-loop corrections are of order $20-30 \%$, with a large uncertainty owing to the uncertainty in the model parameters.

We have focused here on the heavy-to-light meson form factors, but the same Wilson coefficients are also relevant for other processes. The jet-functions calculated here appear in other exclusive processes, such as $B \rightarrow K^{*} \gamma$ and the decays to two light mesons [27]. The one-loop matching corrections to the $\mathrm{SCET}_{\mathrm{I}}$ current operators might become relevant for the study of power corrections to inclusive decay distributions for processes like $B \rightarrow X_{u} \ell \nu$ and $B \rightarrow X_{s} \gamma[28]$.

\section{Acknowledgments}

We thank M. Beneke and D. s. Yang for alerting us to the scheme dependence of the one-loop jet functions and M. Neubert for useful discussions. The work of T.B. and R.J.H. is supported by the Department of Energy under Grant DE-AC02-76SF00515 and by the National Science Foundation under Grant PHY99-07949.

\section{References}

[1] M. Neubert, Phys. Rev. D 49, 3392 (1994) [hep-ph/9311325]. 
[2] I. I. Y. Bigi, M. A. Shifman, N. G. Uraltsev and A. I. Vainshtein, Int. J. Mod. Phys. A 9, 2467 (1994) [hep-ph/9312359].

[3] G. P. Korchemsky and G. Sterman, Phys. Lett. B 340, 96 (1994) [hep-ph/9407344].

[4] M. Beneke, G. Buchalla, M. Neubert and C. T. Sachrajda, Phys. Rev. Lett. 83, 1914 (1999) [hep-ph/9905312].

[5] C. W. Bauer, S. Fleming, D. Pirjol and I. W. Stewart, Phys. Rev. D 63, 114020 (2001) [hep-ph/0011336].

[6] C. W. Bauer, D. Pirjol and I. W. Stewart, Phys. Rev. D 65, 054022 (2002) [hep$\mathrm{ph} / 0109045]$.

[7] J. Chay and C. Kim, Phys. Rev. D 65, 114016 (2002) [hep-ph/0201197].

[8] M. Beneke, A. P. Chapovsky, M. Diehl and T. Feldmann, Nucl. Phys. B 643, 431 (2002) [hep-ph/0206152].

[9] R. J. Hill and M. Neubert, Nucl. Phys. B 657, 229 (2003) [hep-ph/0211018].

[10] M. Beneke and T. Feldmann, Nucl. Phys. B 592, 3 (2001) [hep-ph/0008255].

[11] C. W. Bauer, D. Pirjol and I. W. Stewart, Phys. Rev. D 67, 071502 (2003) [hep$\mathrm{ph} / 0211069]$.

[12] M. Beneke and T. Feldmann, Nucl. Phys. B 685, 249 (2004) [hep-ph/0311335].

[13] B. O. Lange and M. Neubert, Nucl. Phys. B 690, 249 (2004) [hep-ph/0311345].

[14] For a review see: M. Neubert, Phys. Rept. 245 (1994) 259 [hep-ph/9306320].

[15] J. Charles, A. Le Yaouanc, L. Oliver, O. Pene and J. C. Raynal, Phys. Rev. D 60, 014001 (1999) [hep-ph/9812358].

[16] R. J. Hill, T. Becher, S. J. Lee and M. Neubert, JHEP 0407, 081 (2004) [hep-ph/0404217].

[17] M. Beneke, Y. Kiyo and D. s. Yang, Nucl. Phys. B 692, 232 (2004) [hep-ph/0402241].

[18] A. J. Buras and P. H. Weisz, Nucl. Phys. B 333, 66 (1990).

[19] M. J. Dugan and B. Grinstein, Phys. Lett. B 256, 239 (1991).

[20] S. Herrlich and U. Nierste, Nucl. Phys. B 455, 39 (1995) [hep-ph/9412375].

[21] A. V. Manohar, T. Mehen, D. Pirjol and I. W. Stewart, Phys. Lett. B 539, 59 (2002) [hep-ph/0204229].

[22] T. Becher, R. J. Hill and M. Neubert, Phys. Rev. D 69, 054017 (2004) [hep-ph/0308122]. 
[23] T. Becher, R. J. Hill, B. O. Lange and M. Neubert, Phys. Rev. D 69, 034013 (2004) [hep-ph/0309227].

[24] For a review see: J. Collins, Renormalization, (Cambridge Univ. Press, Cambridge, 1984).

[25] A. G. Grozin and M. Neubert, Phys. Rev. D 55, 272 (1997) [hep-ph/9607366].

[26] V. M. Braun, D. Y. Ivanov and G. P. Korchemsky, Phys. Rev. D 69, 034014 (2004) [hep-ph/0309330].

[27] C. W. Bauer, D. Pirjol, I. Z. Rothstein and I. W. Stewart, hep-ph/0401188.

[28] M. Neubert, hep-ph/0408179. 